\title{
Analysis of Stochastic Petri nets with signals
}

\author{
Andrea Marin* Simonetta Balsamo ${ }^{\dagger} \quad$ Peter G. Harrison ${ }^{\ddagger}$
}

July 5, 2012

\begin{abstract}
Product-form models facilitate the efficient analysis of large stochastic models and have been sought after for some three decades. Apart from the dominating work on queueing networks, some product-forms were found for stochastic Petri nets (SPNs) that allow fork-join constructs and for queueing networks extended to include special customers called signals, viz. G-networks. We appeal to the Reversed Compound Agent Theorem (RCAT) to prove new product-form solutions for SPNs in which there are special transitions, the firings of which act in a similar way to signals in G-networks, but which may be generated by synchronised firings (or service completions) and may affect several places simultaneously. We show that SPNs with signals are strict generalisations of G-networks with negative customers, triggers and catastrophes, and illustrate with copious examples.
\end{abstract}

\section{Introduction}

Stochastic modelling has provided powerful methods for the performance evaluation of both hardware and software architectures. More specifically, models based on Continuous Time Markov Chains (CTMCs) play a pivotal role in this context and have been applied successfully to the performance evaluation of various real-world systems (see e.g. [1]). However, it is well-known that even structurally simple models may have a state space with cardinality that makes it infeasible to apply standard techniques in the computation of the stationary (equilibrium) probability distribution of the state. During the past three decades, considerable attention has been devoted to the identification of classes of stochastic models for which the stationary distributions are in product-form. This property essentially decomposes a model $\mathcal{M}$ into a set of $N$ sub-models $\left\{\mathcal{M}_{1}, \ldots, \mathcal{M}_{N}\right\}$, the stationary distributions of each of which can be computed efficiently. At equilibrium, let $\mathbf{m}=\left(m_{1}, \ldots, m_{N}\right)$ denote a state of $\mathcal{M}$, where the component $m_{i}$ is the state of sub-model $\mathcal{M}_{i}, 1 \leq i \leq N$. Then, $\mathcal{M}$ is in product-form if the stationary probability $\pi(\mathbf{m})$ of each state $\mathbf{m}$ is proportional to the product of the stationary probabilities $\pi_{i}\left(\mathbf{m}_{i}\right)$ of each state $m_{i}$ in sub-model $\mathcal{M}_{i}$, i.e:

$$
\pi(\mathbf{m}) \propto \prod_{i=1}^{N} \pi_{i}\left(m_{i}\right) .
$$

*Università Ca' Foscari Venezia, marin@dsi.unive.it

†Università Ca' Foscari Venezia, balsamo@dsi.unive.it

${ }_{\ddagger}^{\ddagger}$ Imperial College, Department of Computing, pgh@doc.ic.ac.uk 
Product-form theory has greatly enhanced the tractability of large stochastic models, largely due to the seminal results proved in queueing theory, such as Jackson's theorem and its multi-class extension, the BCMP theorem $[32,4]$.

Another important class of models for which the underling processes are CTMCs is that of stochastic Petri nets (SPNs). Roughly speaking, the structure of a SPN consists of places, transitions and arcs, the state being given by the numbers of tokens in each place. Arcs connect places to transitions and transitions to places. A state-transition event occurs when a transition fires, after a negative exponentially distributed random time, causing tokens to move from its input places to its output places. SPNs are an important formalism for the performance evaluation of complex systems mainly because they are able to describe, in a natural way, concurrency and synchronization, such as the fork-join construct and parallel computation. In the late 1980s, productform solutions were obtained for SPNs such that synchronisation, batch token movements and resource competition were allowed [31, 8]. In common with Jackson's theorem and the BCMP theorem, these product-forms follow from a set of linear equations that yield the correct parameterisation of associated sub-models, namely, the routing process invariant measures, analogous to the traffic equations of queueing networks.

In the early 1990s, the discovery of a novel product-form model, the Gnetwork, showed that the traffic equations become non-linear when negative customers are introduced $[13,14]$. Differently from the ordinary customers of standard queueing networks, at a negative customer arrival epoch, the queue looses a positive customer if any is present (if the queue is empty, the negative customer simply vanishes). Since this seminal research, a number of new product-form queueing networks with quite sophisticated behaviours have been found, in which the concept of negative customer is generalised to that of a signal, which may act as a trigger that moves a customer from the queue of the arrival (when non-empty) to another queue, chosen probabilistically, or as a catastrophe, which empties the queue. Accessible surveys are given in the books $[22,23]$, the latter featuring some telecommunications applications; see also [7].

In this paper, we appeal to the Reversed Compound Agent Theorem (RCAT) of [28] to derive new product-form solutions for SPN models in which signals are introduced. We first define a set of transitions with a simple semantics that describes signals in a compact way. We then prove that a SPN with such transitions may always be transformed into an equivalent SPN with only standard exponential transitions, but with inhibitor arcs as well. RCAT is a very general result for the derivation of product-forms in compositional models using a modular approach. This is because it is never necessary to consider the CTMC underlying the whole model in order to establish the state distribution at equilibrium. Moreover, RCAT ensures a priori the product-form solution of models in which some components with complicated behaviours (not necessarily specified as SPNs) interact with SPNs having signals - see, for example, [34].

The parameterisation of the sub-models is given by the solution of RCAT's system of rate equations, which are equivalent to the traffic equations in the case of Jackson networks [28]. Differently from G-network signals, SPN signals may be generated by synchronised job completion events, and may affect a set of places chosen probabilistically. Another significant difference from what is known in G-networks is that SPNs with signals may have rate-dependent 
product-form conditions. We prove that the product-form solution holds for models with both triggers (including negative customers) and catastrophes. As one might expect, in SPNs with signals, RCAT's rate equations form, in general, a non-linear system. In the case of G-networks, the existence of a solution to their non-linear traffic equations is considered in [24] by a generic method using Brouwer's fixed point theorem [5]. In a similar fashion, an analogous result on the existence and uniqueness of a solution to RCAT's rate equations is given in [30]. We show that the existence of a solution to the rate equations obtained in this paper follows from the latter. Our method is computationally efficient because it exploits a decomposition of a net into a set of building blocks, the analysis of which can be carried out independently of each other, once the set of rate equations is solved.

Related works. Since they were first introduced in [13, 14, 18], G-networks have proved to be a valuable tool for performance evaluation and optimisation purposes. For instance, in [25], they are used to model a multimedia server system in which customers require "documents" that are transmitted on the shared media. Several other applications of G-networks have been presented in the literature by various authors (see [17, 2, 29, 27]). Following on from the seminal works of $[13,14]$, a number of extensions have been proposed. The aforementioned triggers were introduced in [16] and generalised to the signals studied in [15], which can remove a batch of positive customers from one queue whilst adding a positive one to another. According to [6], we call a signal that completely flushes a node of all its customers at the arrival epoch a catastrophe. Triggers and signals as presented in $[16,15]$ play an important role in the novel results that we introduce in this paper. In fact, as observed in [16], these mechanisms can indeed be useful for representing certain token movements in stochastic Petri nets. Other extensions are important in queueing network theory, particularly those with multiple classes of positive customers or signals $[11,21]$ and the so-called reset customers of [19, 29]. Resets move from one node to another and, on arriving at an empty queue, change the number of enqueued customers from zero to some random value that has a probability distribution that coincides with the steady-state distribution of the number of customers in that queue. Further properties of G-Networks with resets were considered in [20], where the notions of stationary equivalence and flow equivalence were considered and it was shown that, for this class of models, the latter implies the former.

Product-forms in stochastic Petri nets have also received considerable attention, see e.g. $[31,8,35,3]$. One of the interesting aspects of the models studied in these papers is that synchronised token arrivals and departures may lead to rate-dependent product-form conditions. In $[31,8]$ the rate conditions arise from the application of a so-called "rank theorem", in which the rank of a matrix of rates must be checked, whereas in [3], these conditions can be checked in a modular way as a consequence of a structural decomposition of the model. This helps in giving a physical interpretation to conditions that would otherwise be purely algebraic. In relation to other product-form models, we can say first that SPNs with signals generalise G-networks with negative customers, triggers and catastrophes $[14,16,15,10]$. Moreover, they subsume the product-form SPN of [8], without batch token movements. It is worthwhile noting that, differently 
from $[31,8]$, we deal mainly with open models, since triggers may cause token destructions, leading to an empty network in the long-term.

The problem of synchronised arrivals in G-networks has also been addressed in [29] and, more recently, in [9]. The present paper differs from these in that it deals with exact analyses and does not need to introduce a network perturbation in order to derive a product-form, stationary state distribution. Moreover, interpreting the places of a SPN as queues, and the tokens as the network's customers, we show that our approach is also able to model synchronised job completions and the synchronised effects of signals. For instance, we can model a G-network in which a trigger is generated by synchronised job completions in a network of queues, whence it removes simultaneously one customer from each queue belonging to a set chosen probabilistically, and adds a customer to every queue in another set, also chosen probabilistically. As far as we know, in the literature, a product-form solution for this kind of model has never been obtained. In Appendix B, we provide a more technical comparison, in terms of queueing networks, between what SPNs with signals are able to model and what is known from the literature to have a product-form solution.

Structure of the paper. The paper is structured as follows. In Section 2 we introduce, by means of a small example, the aims of the paper. Section 3 briefly recalls the basic notions of SPNs and introduces our notation. In particular, Section 3.1 gives a formal definition of the building blocks used, together with a theorem about the existence of and the expression for their productform stationary state distributions (Theorem 1). In Section 4, we present the product-form analysis of SPNs with signals, specifically with trigger-transitions (Section 4.1) and with catastrophe-transitions (Section 4.2). Concluding remarks are made in Section 5.

\section{Motivating example}

To highlight the key idea of the novel contribution of the paper, we first consider a very simple, illustrative example. The results are generalised and explored more deeply in the following sections. Consider the model shown in Figure 1. Customers (or tokens, in SPN terminology) at place $P_{1}\left(P_{2}\right)$ are introduced as arrivals from transition $T_{A}\left(T_{D}\right)$ at rate $\lambda_{A}\left(\lambda_{D}\right)$; these transitions are essentially Poisson sources. These customers are served (in exponentially distributed time) by transition $T_{E}\left(T_{F}\right)$ at rate $\lambda_{E}\left(\lambda_{F}\right)$. Stochastic Petri nets will be introduced formally in Section 3, but for now we can think of these arrival and service processes as constituting standard exponential queues. However, the introduction of transition $T_{B}$ that simultaneously adds a token to both places $P_{1}$ and $P_{2}$ renders the example indescribable in conventional queueing theory. This is the first peculiarity of SPNs and of our study: the capability of dealing with synchronised arrival events and providing an exact product-form solution.

More importantly for this paper, the behaviour of transition $T_{C}$ is the following: if at the firing epoch both $P_{1}$ and $P_{2}$ are non-empty, then one customer is removed from both of them simultaneously, and one customer is added to place $P_{3}$; if at the firing epoch $P_{1}$ or $P_{2}$ is empty, there is no effect on the model's 


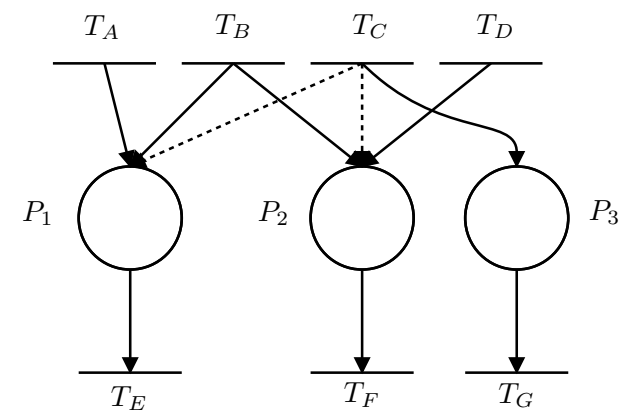

Figure 1: Initial illustrative example.

state. If the following rate-condition is satisfied:

$$
\lambda_{B} \lambda_{E} \lambda_{F}=\lambda_{C} \lambda_{A} \lambda_{D}
$$

then the model is in product-form, and the equilibrium probability function is:

$$
\pi\left(n_{1}, n_{2}, n_{3}\right) \propto\left(\frac{\lambda_{A}}{\lambda_{E}}\right)^{n_{1}}\left(\frac{\lambda_{C}}{\lambda_{F}}\right)^{n_{2}}\left(\frac{\lambda_{B}}{\lambda_{G}}\right)^{n_{3}}
$$

where $\lambda_{G}$ is the rate of transition $T_{G}$, and $n_{1}, n_{2}, n_{3}$ are the numbers of customers in $P_{1}, P_{2}, P_{3}$, respectively. It is worthy of note that if we modify the model such that transition $T_{G}$ is removed and $P_{3}$ is the input place for $T_{B}$, then the model is unconditionally in product-form. In the paper we show how this follows from RCAT's rate equations. Rate dependent product-form conditions are very important in stochastic Petri nets, as initially shown in [8] and, more recently, in $[26,35,3]$. We add to this topic by giving a compositional interpretation of such conditions in terms of our building block basic structures.

\section{Stochastic Petri nets}

In this section, we give the notation we use to describe SPNs and introduce the special class of SPNs that we call building blocks. A stochastic Petri-net is a 7-tuple, $\operatorname{SPN}=\left(\mathcal{P}, \mathcal{T}, \chi(\cdot), \mathbf{I}(\cdot), \mathbf{H}(\cdot), \mathbf{O}(\cdot), \mathbf{m}_{0}\right)$, where:

- $\mathcal{P}=\left\{P_{1}, \ldots, P_{N}\right\}$ is a set of $N$ places,

- $\mathcal{T}=\left\{T_{1}, \ldots, T_{M}\right\}$ is a set of $M$ transitions,

- $\chi: \mathcal{T} \rightarrow \mathbb{R}^{+}$is a positive valued function that associates a firing rate with every transition; we usually write $\chi_{i}$ as an abbreviation for $\chi\left(T_{i}\right)$,

- $\mathbf{I}: \mathcal{T} \rightarrow \mathbb{N}^{N}$ associates an input vector of places with every transition,

- $\mathbf{H}: \mathcal{T} \rightarrow \mathbb{N}^{N}$ associates an inhibitor vector of places with every transition,

- $\mathbf{O}: \mathcal{T} \rightarrow \mathbb{N}^{N}$ associates an output vector of places with every transition,

- $\mathbf{m}_{0} \in \mathbb{N}^{N}$ is the initial marking. 
A state $\mathbf{m} \in \mathbb{N}^{N}$ of the model is called a marking and represents the numbers $m_{i}$ of tokens in each place $P_{i}, i=1, \ldots, N$. A transition $T_{i}$ is enabled by $\mathbf{m}$ if $\left(\mathbf{m}-\mathbf{I}\left(t_{i}\right)\right)$ and $\left(\mathbf{H}\left(t_{i}\right)-\mathbf{m}\right)$ have no negative components. We write $\mathbf{v} \geq \mathbf{v}^{\prime}$ to denote $v_{i} \geq v_{i}^{\prime}$ for $i=1, \ldots, N$, and similarly for the other arithmetic inequalities. Hence, $T_{i}$ is enabled when $\mathbf{m} \geq \mathbf{I}\left(t_{i}\right)$ and $\mathbf{H}\left(t_{i}\right) \geq \mathbf{m}$. An enabled transition $T_{i}$ fires after an exponentially distributed random time with rate $\chi_{i}$. In this case, the new state $\mathbf{m}^{\prime}$ is $\mathbf{m}-\mathbf{I}\left(T_{i}\right)+\mathbf{O}\left(T_{i}\right)$. The net is called ordinary if the input and output vector domains are $\{0,1\}^{N}$, i.e. all their components are either 0 or 1.

Graphically, we draw places as circles and transitions as solid bars. If the $j$-th component of $\mathbf{I}\left(T_{i}\right)$ (respectively $\mathbf{O}\left(T_{i}\right)$ ) is $k>0$ then we draw an arc from $P_{j}$ (respectively $T_{i}$ ) to $T_{i}$ (respectively $P_{j}$ ) and we label it with $k$ (for ordinary nets we omit the labels, which would all be 1).

The reachability set $R S\left(\mathbf{m}_{0}\right)$ is the set of all possible states of the net, given the initial marking $\mathbf{m}_{0}$. In general, the problem of determining the reachability set of a SPN is exponential in space. The reachability graph is a graph whose nodes are the states of the reachability set, in which there is an arc from node $\mathbf{m}^{\prime}$ to $\mathbf{m}^{\prime \prime}$ if there exists a transition $T$ such that $\mathbf{m}^{\prime \prime}=\mathbf{m}^{\prime}-\mathbf{I}(T)+\mathbf{O}(T)$. The incidence matrix $\mathbf{A}$ of a SPN is an $M \times N$ matrix, row $i$ of which is defined as $\mathbf{O}\left(T_{i}\right)-\mathbf{I}\left(T_{i}\right)$. The reachability graph can be either finite or infinite and from it, the continuous time Markov chain (CTMC) underlying the SPN model can be derived simply (either lazily or in a parameterised way if the state space is infinite). Henceforth, we consider models whose underlying CTMCs are ergodic and so admit a unique, equilibrium, state probability distribution. Calculating this can be a difficult computational task because of the state space explosion problem, which causes even a structurally small net to have a reachability set with high cardinality. In such cases, solution of the global balance equations rapidly becomes numerically intractable.

Some structural properties can be decided by analysis of the incidence matrix and, in particular, T-invariants play an important role. They are defined as follows:

Definition 1 (T-invariant and support) Given a SPN with incidence matrix $\mathbf{A}$, a vector $\mathbf{X}=\left(x_{1}, \ldots, x_{M}\right) \in \mathbb{N}^{M}$ is a T-invariant if $\mathbf{A}^{T} \mathbf{X}=\mathbf{0}$.

The support $\|\mathbf{X}\|$ of a T-invariant $\mathbf{X}$ is the set of transitions corresponding to the non-zero entries of $\mathbf{X}$, i.e. those transitions $T_{i}$ for which $x_{i} \neq 0 . A T$ invariant $\mathbf{X}$ is minimal if there is no other T-invariant $\mathbf{X}^{\prime}$ such that $x_{i}^{\prime} \leq x_{i}$ for all $i=1, \ldots, M$. A support of a T-invariant is minimal if no proper non-empty subset of the support is also a support. The minimal support T-invariant is the minimal T-invariant with minimal support.

Definition 2 (Closed T-invariant) A T-invariant is closed if for every transition $T$ of the support there exists another transition with the input vector identical to the output vector of $T$ as well one whose output vector is identical to the input vector of $T$.

\subsection{SPN building blocks (BBs)}

In general, a building block consists of a set of places $P_{1}, \ldots P_{N}$, a set $\mathcal{T}_{I}$ of input transitions whose input vectors are null (i.e. equal to $\mathbf{0}=(0, \ldots, 0)$ ), and 
a set $\mathcal{T}_{O}$ of output transitions whose output vectors are null. Note that the set of all transitions in a $\mathrm{SPN}, \mathcal{T}=\mathcal{T}_{I} \cup \mathcal{T}_{O}$ and that $\mathcal{T}_{I} \cap \mathcal{T}_{O}=\emptyset$. To simplify the notation, we use $T_{y}\left(T_{y}^{\prime}\right)$ to denote an input (output) transition, where $y$ is the set of place-indices of the non-zero components in the output (input) vector of $T_{y}\left(T_{y}^{\prime}\right)$. For example, transition $T_{23}\left(T_{23}^{\prime}\right)$ in the net of Figure 2 is the transition that produces (consumes) tokens at places $P_{2}$ and $P_{3}$. All the arcs have multiplicity 1 . The following definition gives the main restriction on the structure of $\mathrm{BBs}$, that for each input transition $T_{y}$ there must exist an output transition $T_{y}^{\prime}$ whose input vector is equal to the output vector of $T_{y}$.

Definition 3 Two places $P_{i}, P_{j} \in \mathcal{P}, 1 \leq i, j \leq N$, are directly connected, written $P_{i} \sim P_{j}$, if there exists a transition $T \in \mathcal{T}$ such that the components $\mathbf{I}(T)_{i} \neq 0$ and $\mathbf{I}(T)_{j} \neq 0$ or $\mathbf{O}(T)_{i} \neq 0$ and $\mathbf{O}(T)_{j} \neq 0$. Let $\sim^{*}$ be the transitive closure of $\sim P_{i}$ and $P_{j} \in \mathcal{P}$ are connected if $P_{i} \sim^{*} P_{j}$.

Definition 4 (Building block (BB)) Given an ordinary, connected SPN S with set of transitions $\mathcal{T}$ and set of $N$ places $\mathcal{P}$, then $S$ is a building block if it satisfies the following conditions:

1. For all $T \in \mathcal{T}$, either $\mathbf{O}(T)=\mathbf{0}$ or $\mathbf{I}(T)=\mathbf{0}$.

2. For each $T \in \mathcal{T}_{I}$, there exists $T^{\prime} \in \mathcal{T}_{O}$ such that $\mathbf{O}(T)=\mathbf{I}\left(T^{\prime}\right)$ and conversely.

3. Each pair of places $P_{i}$ and $P_{j}$ in the $B B$ are connected.

Condition 1 requires that all the transitions are either input or output transitions, while Condition 2 states that if there exists an input transition $T_{y}$ feeding tokens to a subset of places $y$, then there must be a corresponding output transition $T_{y}^{\prime}$ that consumes the tokens from the same subset $y$. Note that Condition 2 is the structural condition for the product-form class of models defined by Coleman, Henderson et al. in [8].

Figure 2 illustrates an example of a BB consisting of 3 places $\mathcal{P}=\left\{P_{1}, P_{2}, P_{3}\right\}$, 3 input transitions $\mathcal{T}_{I}=\left\{T_{3}, T_{23}, T_{12}\right\}$ and 3 output transitions $\mathcal{T}_{O}=\left\{T_{3}^{\prime}, T_{23}^{\prime}, T_{12}^{\prime}\right\}$.

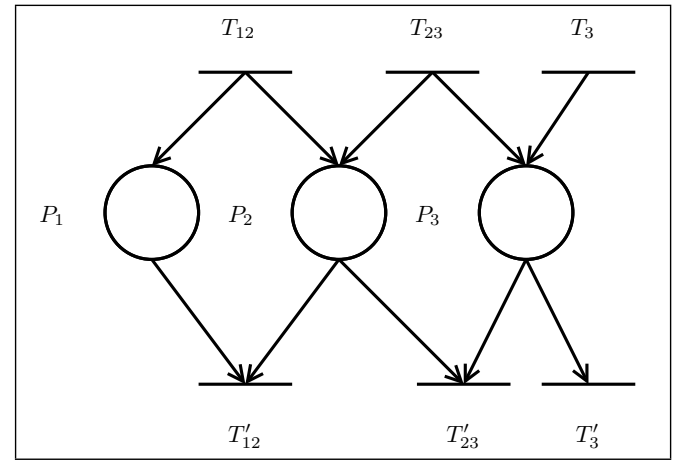

Figure 2: Example of a building block.

Note that if two or more input (output) transitions have the same output (input) vector, we can fuse them in one transition whose rate is the sum of the 
rates of the original transitions. Therefore, without loss of generality, we assume that all the input (output) transitions have different output (input) vectors. For example, given the $\mathrm{BB}$ of Figure 3 , whose transitions $T_{a 12}^{\prime}$ and $T_{b 12}^{\prime}$ share the same input vector, we can consider the equivalent (in terms of steady-state distribution) BB depicted by Figure 2, in which the rate of transition $T_{12}^{\prime}$ is the sum of the rates of transitions $T_{a 12}^{\prime}$ and $T_{b 12}^{\prime}$.

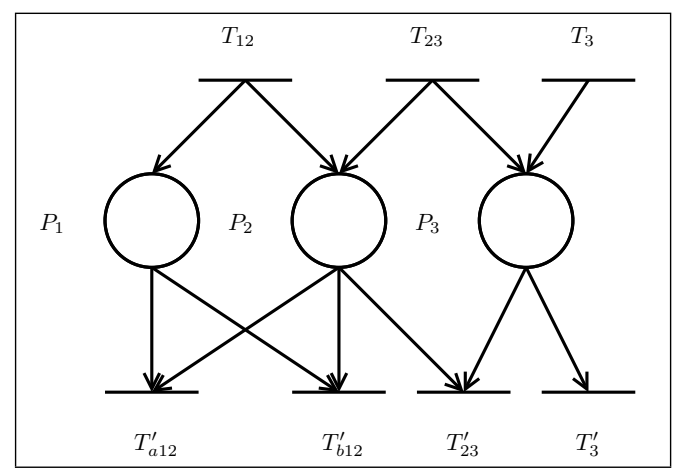

Figure 3: A building block with output transitions having the same input vector.

\subsection{Product-forms for BBs}

In [3] it is shown that an arbitrary BB has product-form solution under a certain set of rate-dependent conditions. Theorem 1 enunciates this result. For compatibility with conventional queueing network notation, we write $\chi_{y}=\lambda_{y}$ and $\chi_{y}^{\prime}=\mu_{y}$ to specify the rates of the input and output transitions, respectively, where $y \in 2^{\{1, \ldots, N\}} \backslash \emptyset$.

Theorem 1 Consider a BBS with $N$ places and let $\mathcal{N} \subseteq 2^{\{1, \ldots, N\}} \backslash \emptyset$. Let $\rho_{y}=\lambda_{y} / \mu_{y}$ for positive, real variables $\lambda_{y}, \mu_{y}$ and for $T_{y}, T_{y}^{\prime} \in \mathcal{T},|y| \geq 1$. Then if the following system of equations has a unique solution $\rho_{i}, 1 \leq i \leq N$,

$$
\begin{cases}\rho_{y}=\prod_{i \in y} \rho_{i} & \forall y: T_{y}, T_{y}^{\prime} \in \mathcal{T} \wedge|y|>1 \\ \rho_{i}=\frac{\lambda_{i}}{\mu_{i}} & \forall i: T_{i}, T_{i}^{\prime} \in \mathcal{T}, 1 \leq i \leq N\end{cases}
$$

then the net's balance equations - and hence stationary probabilities when they exist - have product-form solution:

$$
\pi\left(m_{1}, \ldots, m_{N}\right) \propto \prod_{i=1}^{N} \rho_{i}^{m_{i}}
$$

Note that System (1) has $N$ unknowns $\rho_{1}, \ldots, \rho_{N}$. However, for each $1 \leq i \leq N$ such that $T_{i}, T_{i}^{\prime} \in \mathcal{T}$ we straightforwardly have $\rho_{i}=\lambda_{i} / \mu_{i}$. Moreover, the system can be solved as a linear system by taking logarithms on both sides.

Example 1 Consider the BB of Figure 2. The Equations (1) become:

$$
\left\{\begin{array}{l}
\rho_{3}=\lambda_{3} / \mu_{3} \\
\rho_{12}=\lambda_{12} / \mu_{12}=\rho_{1} \rho_{2} \\
\rho_{23}=\lambda_{23} / \mu_{23}=\rho_{2} \rho_{3}
\end{array}\right.
$$


from which we immediately obtain:

$$
\rho_{2}=\lambda_{23} \mu_{3} /\left(\mu_{23} \lambda_{3}\right) \quad \rho_{1}=\lambda_{12} \mu_{23} \lambda_{3} /\left(\mu_{12} \lambda_{23} \mu_{3}\right)
$$

and hence an unconditioned product-form:

$$
\pi\left(n_{1}, n_{2}, n_{3}\right) \propto\left(\frac{\lambda_{12} \mu_{23} \lambda_{3}}{\mu_{12} \lambda_{23} \mu_{3}}\right)^{n_{1}}\left(\frac{\lambda_{23} \mu_{3}}{\mu_{23} \lambda_{3}}\right)^{n_{2}}\left(\frac{\lambda_{3}}{\mu_{3}}\right)^{n_{3}}
$$

Now consider the BB of Figure 4 that differs from that of Figure 2 because of transitions $T_{1}$ and $T_{1}^{\prime}$. In this case Equations (1) are satisfied if

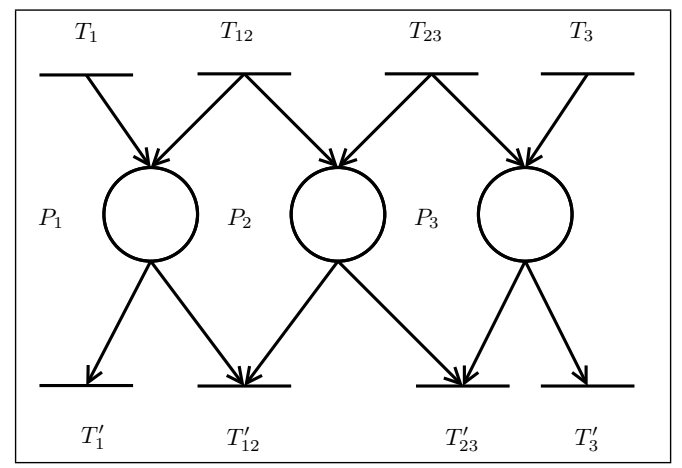

Figure 4: A building block with rate-dependent product-form conditions.

$$
\rho_{2}=\frac{\rho_{12}}{\rho_{1}}=\frac{\rho_{23}}{\rho_{3}}
$$

i.e.:

$$
\lambda_{12} \mu_{1} \lambda_{3} \mu_{23}=\mu_{3} \lambda_{23} \mu_{12} \lambda_{1}
$$

Under this assumption, the product-form expression is given by Equation (2).

We now show that if a composition of BBs, in which the output transition of a component may be the input transition of another, has a steady-state solution, then this has a product-form. The proof is a consequence of the fact that each building block satisfies the conditions of RCAT if we consider the statetransitions (in the underlying Markov process) corresponding to input SPNtransitions as passive and those corresponding to output SPN-transitions as active. We need the following lemma:

Lemma 1 In a product-form BB that satisfies the conditions of Theorem 1, the reversed rate of every output transition labelled $T_{y}^{\prime}$ is $\lambda_{y}$, i.e., the rate of the corresponding input transition, and the reversed rate of every input transition labelled $T_{y}$ is $\mu_{y}$, i.e., the rate of the corresponding output transition.

Proof By hypothesis, the stationary distribution of the BB is given by Equation (2). Let $\mathbf{I}\left(T_{y}^{\prime}\right)$ be the input vector of $T_{y}^{\prime} \in \mathcal{T}_{O}$ and let $\mathbf{m}$ be a reachable marking that enables $T_{y}^{\prime}$, i.e., $\mathbf{m}>\mathbf{I}\left(T_{y}^{\prime}\right)$. The reversed rate $\overline{\mu_{y}}$ of $T_{y}^{\prime}$ is:

$$
\overline{\mu_{y}}=\frac{\pi(\mathbf{m})}{\pi\left(\mathbf{m}-\mathbf{I}\left(T_{y}^{\prime}\right)\right)} \mu_{y}=\mu_{y} \prod_{i \in y} \rho_{i} .
$$


Since the $\rho_{i}$ are the solutions of equations (1), we have $\overline{\mu_{y}}=\rho_{y} \mu_{y}=\lambda_{y}$, as required. The result for the reversed rates of the input transitions is similar.

Alternatively, the same result can be written down by noting that the BB is a reversible Markov process [33].

Proposition 1 Suppose that in a composition of BBs,

- each output transition of any BB is either the input transition of another $B B$ or else an output transition of the composition (an "external departure");

- conversely, each input transition of any BB is either the output transition of another BB or else an input transition of the composition (an "external arrival").

Then any irreducible subset of the markings of the SPN defined by the composition of the BBs has product-form.

Proof In the underlying Markov process of each component-BB, take:

- the state-transitions corresponding to SPN-transitions $T_{y} \in \mathcal{T}_{I}$ to be passive and labelled by $t_{y}$;

- the state-transitions corresponding to SPN-transitions $T_{y}^{\prime} \in \mathcal{T}_{O}$ to be active and labelled by $t_{y}^{\prime}$.

Then RCAT's conditions apply to irreducible subsets of the composition (the third condition holds by Lemma 1), which thereby has product-form [28].

\section{Product-forms for ordinary SPNs with signals}

Compositions of BBs that admit a stationary distribution have product-form solutions by Proposition 1. Moreover, when such a composition forms a closed SPN, then the product-form belongs to the class of ordinary product-form SPNs of $[31,8,26]$. We now show that Theorem 1 and Proposition 1 not only generalise the product-form result in a uniform way from closed nets to open ones (with, in general, rate-dependent conditions), but also allows one to derive product-form solutions for more complex models that are closely related to G-networks and their variants. In fact, we can apply the idea of signals (i.e. negative customers or triggered customer movements [16]), inherited from the G-network literature, to derive further product-form solutions in ordinary SPNs. We obtain a model that may be seen either as a SPN with G-network-style triggers, or as a Gnetwork in which synchronised job completions, arrivals, or triggers may occur.

\subsection{SPNs with triggers}

A negative customer that arrives at a non-empty queue deletes, or kills, one of the customers in that queue or in service; at an empty queue, the negative customer disappears, i.e. has no effect, see [14]. A trigger is a generalisation of a negative customer, introduced in [16], which vanishes on arrival at an empty queue or else transfers one of the customers present to a target queue, 
usually chosen probabilistically. In the special case that a trigger transfers a customer outside the whole network, the trigger degenerates into a regular negative customer - hence triggers are a true generalisation.

In [29], the notion of such triggers was extended to include negative triggers - those defined in the previous paragraph now being distinguished by the name positive triggers. A negative trigger is the same as a positive trigger except that another negative trigger is transferred rather than a customer. Notice that if the transferred negative trigger is not a regular negative customer, the chain will continue until an empty queue is encountered or a negative customer is eventually transferred. It is shown further how cycles of negative triggers can model catastrophes, where whole queues can be cleared on the arrival of a negative trigger.

In this section, we show that an output transition of a building block may generate trigger-tokens that have the same effect as triggers in G-networks and that, under a certain set of conditions, the product-form is preserved. Differently from G-networks, however, trigger-token arrivals may have synchronised effects, i.e. delete or move tokens from more than one place belonging to the same BB simultaneously. Similarly, they may be generated by a transition that synchronises an arbitrary number of places belonging to the same building block. Whilst we use a terminology that refers to trigger-tokens and trigger-transitions for compatibility with the body of G-network literature, the semantics presented next does not require an extension of the SPN definition given in Section 3.1, as shown by Lemma 2 .

\subsubsection{Semantics}

We introduce into the Petri net formalism a new type of transition, namely the trigger-transition, the firings of which cause a trigger-token-arrival at the some places, and a token-arrival at others. Let $T^{\dagger}$ be a trigger-transition, which is characterised by an input vector identical to that of ordinary transitions and by two output vectors $\mathbf{O}^{-}\left(T^{\dagger}\right)$ and $\mathbf{O}\left(T^{\dagger}\right)$. The trigger-transition is enabled by a marking $\mathbf{m}$ according to the same rule given for ordinary transitions, i.e. $\mathbf{m}-\mathbf{I}\left(T^{\dagger}\right) \geq \mathbf{0}$, but the state entered after its firing is:

- $\mathbf{m}-\mathbf{I}\left(T^{\dagger}\right)-\mathbf{O}^{-}\left(T^{\dagger}\right)+\mathbf{O}\left(T^{\dagger}\right)$ if $\mathbf{m}-\mathbf{O}^{-}\left(T^{\dagger}\right) \geq \mathbf{0}$

- $\mathbf{m}-\mathbf{I}\left(T^{\dagger}\right)$ otherwise,

where we assume that the supports of $\mathbf{I}\left(T^{\dagger}\right)$ and $\mathbf{O}^{-}\left(T^{\dagger}\right)$ are disjoint. A triggertransition is indicated graphically by using dotted arcs between it and the places specified for token removal by non-zero components in $\mathbf{O}^{-}$; the places that receive the transferred tokens, specified by $\mathbf{O}$, are connected by normal lines. This is illustrated in Figure 5, where transition $T_{E}$ generates a trigger-token for places $P_{4}$ and $P_{5}$. If these places are both non-empty, then a positive token is added to place $P_{6}$ and one token is removed from each of $P_{4}$ and $P_{5}$. Rather than an extension to the Petri net formalism (which it is not), this structure is employed as a convenient means to investigate new product-forms. In fact we have the following result.

Proposition 2 Given a SPN $S$ with trigger-transitions, there exists a stochastically equivalent, standard $S P N S^{*}$, i.e. a SPN belonging to the class given by Definition 3 in Section 3.1 that has the same underlying Markov process. 


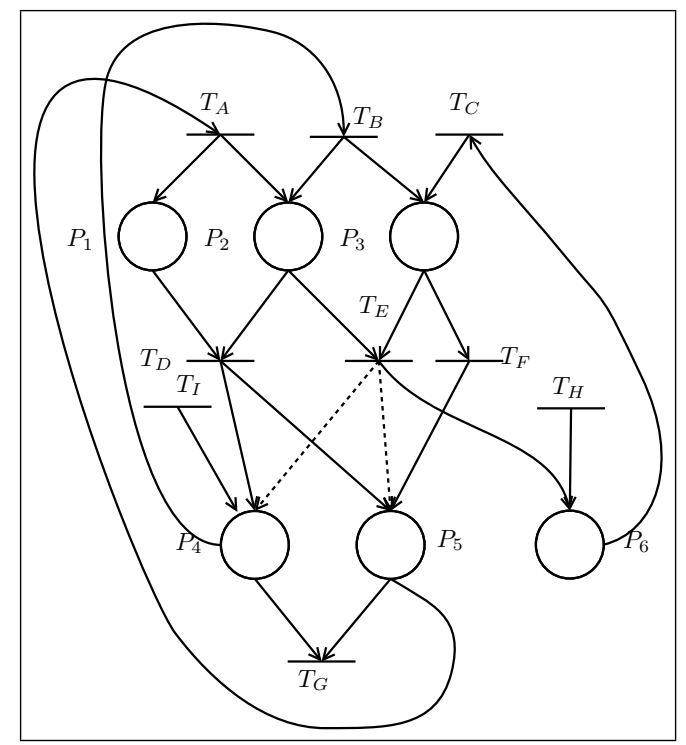

Figure 5: A SPN with negative transitions.

Proof. For every trigger-transition $T^{\dagger}$ of $S$, let $w$ be the set of non-zero components of $\mathbf{O}^{-}\left(T^{\dagger}\right)$ and $\mathcal{W}=2^{w}$. Let $\mathbf{1}_{s}$, with $s \in \mathcal{W}$, be a vector whose component $i$ is 1 if $i \in s$ and 0 otherwise. Then, in $S^{*}$, transition $T^{\dagger}$ is replaced by a set of transitions $\mathcal{T}\left(T^{\dagger}\right)=\left\{T_{z}, z \in \mathcal{W}\right\}$ with the same rate of $T^{\dagger}$ such that if $z \neq w$, then $\mathbf{I}\left(T_{z}\right)=\mathbf{I}\left(T^{\dagger}\right)+\mathbf{1}_{z}, \mathbf{H}\left(T_{z}\right)=\mathbf{1}_{w \backslash z}$, and $\mathbf{O}\left(T_{z}\right)=\mathbf{1}_{z}$. If $z=w$, then $\mathbf{I}\left(T_{w}\right)=\mathbf{I}\left(T^{\dagger}\right)+\mathbf{1}_{w}, \mathbf{H}\left(T_{w}\right)=\mathbf{0}$ and $\mathbf{O}\left(T_{w}\right)=\mathbf{O}\left(T^{\dagger}\right)$. The Markov processes underlying $S$ and $S^{*}$ are now easily seen to be identical.

\subsubsection{Analysis}

We now give the product-form, together with its conditions, for SPNs with trigger-transitions. First, we extend the connectivity relation $\sim^{*}$ introduced in Definition 3 in order to incorporate trigger-transitions.

Definition 5 In a SPN with trigger-transitions, we say that two places $P_{i}$ and $P_{j} \in \mathcal{P}$ are directly connected with or without triggers, written $P_{i} \sim_{t} P_{j}$, if $P_{i} \sim P_{j}$ or there exists a trigger-transition $T^{\dagger}$ such that $\mathbf{O}^{-}\left(T^{\dagger}\right)_{i} \neq 0$ and $\mathbf{O}^{-}\left(T^{\dagger}\right)_{j} \neq 0$. We define the equivalence relation $\sim_{t}^{*}$ to be the transitive closure of $\sim_{t}$, and say that $P_{i}$ and $P_{j}$ are connected (with or without triggers) if $P_{i} \sim_{t}^{*}$ $P_{j}$.

The equivalence classes of $\sim_{t}^{*}$ are labelled $\mathcal{C}^{(b)}, 1 \leq b \leq B$ where $B>0$ is the number of equivalence classes. We define $\sigma(\mathbf{v})$ to be the set of indices of the non-zero components of the vector $\mathbf{v}$.

Definition 6 Given a SPN $S$ with trigger-transitions and $\sim_{t}^{*}$-equivalence classes $\mathcal{C}^{(b)}, 1 \leq b \leq B$, the net decomposition of $S$ is the set of SPNs $S^{d e c}=$ $\left\{B B^{(b)}, \overline{1} \leq b \leq B\right\}$, in which the set of places of $B B^{(b)}$ is $\mathcal{C}^{(b)}$ and the transitions of each $B B^{(b)}$ in $S^{\text {dec }}$ are given by the following, where the generic transition $T \in \mathcal{T}_{S}$ with rate $\chi$ is in $S$ : 
- Let $y=\sigma(\mathbf{I}(T)) \subseteq \mathcal{C}^{(b)}, y \neq \emptyset$ for some $b, 1 \leq b \leq B$. Then $B B^{(b)}$ has an output transition $T_{y}^{\prime}$ with $\mathbf{I}\left(T_{y}^{\prime}\right)=\mathbf{I}(T)$ and rate $\chi$

- If $T$ is ordinary, let $w=\sigma(\mathbf{O}(T)) \subseteq \mathcal{C}^{(c)}, w \neq \emptyset$ for some $c, 1 \leq c \leq B$. Then $B B^{(c)}$ has an input transition $T_{w}$ with $\mathbf{O}\left(T_{w}\right)=\mathbf{O}(T)$ and rate equal to $\chi$ if $\mathbf{I}(T)=\mathbf{0}$ or equal to the reversed rate of $T_{y}$ in $B B^{(b)}$, containing the places $y=\sigma(\mathbf{I}(T))$, otherwise.

- If $T$ is a trigger-transition, let $u=\sigma\left(\mathbf{O}^{-}(T)\right) \subseteq \mathcal{C}^{(d)}, u \neq \emptyset$ for some $d, 1 \leq d \leq B$. Then $B B^{(d)}$ has an output transition $T_{u}^{\prime}$ with $\mathbf{I}\left(T_{u}^{\prime}\right)=$ $\mathbf{O}^{-}(T)$ and rate equal to $\chi$ if $\mathbf{I}(T)=\mathbf{0}$ or equal to the reversed rate of $T_{y}^{\prime}$ in $B B^{(b)}$, containing the places $y=\sigma(\mathbf{I}(T))$, otherwise.

- If $T$ is a trigger-transition, let $z=\sigma(\mathbf{O}(T)) \subseteq \mathcal{C}^{(e)}, z \neq \emptyset$ for some $e, 1 \leq e \leq B$. Then $B B^{(e)}$ has an input transition $T_{z}$ with $\mathbf{O}\left(T_{z}\right)=\mathbf{O}(T)$ and rate equal to the reversed rate of $T_{u}^{\prime}$ in $B B^{(d)}$, containing the places $u=\sigma\left(\mathbf{O}^{-}(T)\right)$.

$A$ net decomposition is said to be well-formed if $B B^{(i)}$ is a $B B$ for every $i=$ $1, \ldots, B$.

\section{Remarks}

1. The choice of certain rates as the reversed rates of other transitions requires the solution of the rate equations of RCAT, which can certainly be applied when the net decomposition is well-formed.

2. It is common for different transitions in a SPN to share the same input or output vectors and this may cause conflicts on the labels of the input/output transitions in the BBs. This may also happen with triggertransitions. BB transitions with the same input/output vectors are therefore distinguished by adding an additional label. Suppose that transitions $T_{A}$ and $T_{B}$ have the same output vector and $y=\sigma\left(\mathbf{O}\left(T_{A}\right)\right)=\sigma\left(\mathbf{O}\left(T_{B}\right)\right)$. The corresponding input transitions of the associated $\mathrm{BB}$ are then named $T_{a y}$ and $T_{b y}$, as shown in Figure 3. The analysis can now be carried out as explained in Section 3.1.

Theorem 2 A SPN with well-formed decomposition $S^{d e c}=\left\{B B^{(1)}, \ldots, B B^{(B)}\right\}$ has product-form solution:

$$
\pi(\mathbf{m}) \propto \prod_{b=1}^{B} \pi_{b}(\mathbf{m})
$$

where $\pi_{b}$ is an un-normalised product-form solution of $B B^{(b)}$, with rates set according to Definition 6, if each $B B^{(b)}$ satisfies the rate conditions of Theorem 1.

Proof. First, suppose $T$ is an ordinary transition of $S$ and, according to Definition 6 , let $T_{y}$ and $T_{w}^{\prime}$ be the corresponding input and output transitions of $B B^{\left(b_{1}\right)}$ and $B B^{\left(b_{2}\right)}$, respectively, $1 \leq b_{1} \neq b_{2} \leq B$. Then, in the underlying Markov processes, $T_{y}$ and $T_{w}^{\prime}$ are modelled by passive and active transitions respectively, the rate of $T_{w}^{\prime}$ being the given rate of $T$. By Proposition 1, if the 
RCAT product-form exists, then the rate of $T_{y}\left(x_{y}\right)$ must be the reversed rate of $T_{w}^{\prime}$ in $B B^{\left(b_{1}\right)}$, i.e. the rate of $T_{w}$ which, according to Definition 6 , is either a passive rate variable or the rate of an input transition, assumed constant.

The case of trigger-transitions is more complicated and RCAT must be applied twice, as in [29], for example. Let $T^{\dagger}$ be a trigger-transition, and $T_{y}^{\prime}$ in $B B^{\left(b_{1}\right)}, T_{u}^{\prime}$ in $B B^{\left(b_{2}\right)}$, and $T_{z}$ in $B B^{(3)}$ be the transitions in the decomposition corresponding to $\mathbf{I}\left(T^{\dagger}\right), \mathbf{O}^{-}\left(T^{\dagger}\right)$ and $\mathbf{O}\left(T^{\dagger}\right)$, respectively, $1 \leq b_{1}, b_{2}, b_{3} \leq B$. First consider the synchronisation between $B B^{\left(b_{1}\right)}$ and $B B^{\left(b_{2}\right)}$. In the underlying Markov processes, we model $T_{y}^{\prime}$ by an active transition type, whose rate is that of $T^{\dagger}$, and $T_{u}^{\prime}$ by a passive transition type. Note that $T_{u}^{\prime}$ is not outgoing from states of $B B^{\left(b_{2}\right)}$ in which at least one of the input places is empty. However, to avoid blocking, consistent with G-network semantics, $B B^{\left(b_{1}\right)}$ must still be able to change its state due to the firing of $T^{\dagger}\left(T_{y}^{\prime}\right)$ but the trigger has no effect on $B B^{\left(b_{2}\right)}$. This behaviour is modelled by adding passive self-loops (with the same type as $T_{u}^{\prime}$ ) on the states where $T_{u}^{\prime}$ is not outgoing. Now RCAT's structural conditions are satisfied and the rate of $T_{u}^{\prime}$ is the reversed rate of $T_{y}^{\prime}$, which is constant by Lemma 1 .

Finally, we consider the joint process consisting of $B B^{\left(b_{1}\right)}$ and $B B^{\left(b_{2}\right)}$, just proved to be in product-form, synchronised with $B B^{\left(b_{3}\right)}$ by the joint, active firing of $T_{y}^{\prime}, T_{u}^{\prime}$ with the passive $T_{z}\left(\right.$ in $\left.B B^{\left(b_{3}\right)}\right)$. Every state of $B B^{\left(b_{3}\right)}$ has an outgoing, passive transition corresponding to $T_{z}$ and every state of the joint process $\left(B B^{\left(b_{1}\right)}\right.$ and $\left.B B^{\left(b_{2}\right)}\right)$ has an incoming, active transition corresponding to $T_{y}^{\prime}$. Hence RCAT's structural conditions are again satisfied. The reversed rates of these active transitions are the reversed rates of the passive $T_{u}^{\prime}$ in $B B^{\left(b_{2}\right)}$, which are constant by Lemma 1 . This is the rate that must be assigned to the passive transitions $T_{z}$ to compute the product-form.

\subsubsection{Applications}

Theorem 2 can be applied to derive both rate-independent and rate-dependent, product-form conditions and equilibrium probability distributions for the markings of highly non-trivial nets. We now consider some examples.

Example 2 (Open net with rate-dependent product-form condition) We first consider the SPN of Figure 5. Although this paper does not consider the problem of deciding the ergodicity of the Markov process underlying the $\mathrm{SPN}$, we show that the net is live, and that given an arbitrary state $\mathbf{m}$ there exists a sequence of firings that takes the net back to $\mathbf{m}$, viz:

$$
T_{H} \rightarrow T_{C} \rightarrow T_{F} \rightarrow T_{A} \rightarrow T_{D} \rightarrow T_{G}
$$

In order to show that the net is live, and that all the transitions are covered by a T-invariant, we note that we have the following cycles. Suppose that $m_{4}=0$ or $m_{5}=0$ in the initial marking $\mathbf{m}$. Then the firing sequence $T_{I} \rightarrow T_{B} \rightarrow T_{E}$ returns the net to marking $\mathbf{m}$. Now suppose $m_{4}>0$ and $m_{5}>0$. Then the firing sequence

$$
T_{I} \rightarrow T_{B} \rightarrow T_{E} \rightarrow T_{C} \rightarrow T_{F} \rightarrow T_{A} \rightarrow T_{D}
$$

defines a cycle. 
The first step of the stochastic analysis consists of the net decomposition, which is shown in Figure 6 and is well-formed. In the net of Figure 5, let the rate of transition $T_{\alpha}$ be $\chi_{\alpha}, \alpha=A, \ldots, I$; these rates are attached to each transition name in Figure 6, which also shows the rates in the BBs used in Theorem 2. The system of rate equations for the net follows directly from Theorem 2 and

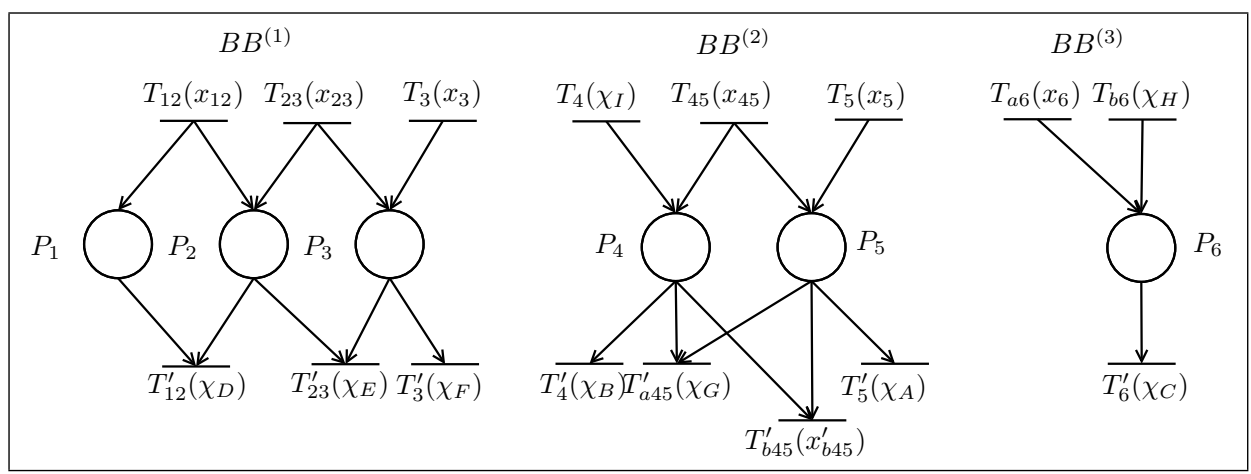

Figure 6: Decomposition of the net of Figure 5.

Lemma 1 as:

$$
\left\{\begin{array}{l}
x_{3}=x_{45}=x_{12}=x_{5}=x_{6}+\chi_{H} \\
x_{b 45}^{\prime}=x_{23}=\chi_{I} \\
x_{6}=x_{45} \frac{x_{b 45}^{\prime}}{\chi_{G}+x_{45^{\prime}}}
\end{array}\right.
$$

which, although non-linear, are easily solved to yield:

$$
\left\{\begin{array}{l}
x_{3}=x_{45}=x_{12}=x_{5}=\frac{\chi_{H}\left(\chi_{I}+\chi_{G}\right)}{\chi_{G}} \\
x_{b 45}^{\prime}=x_{23}=\chi_{I} \\
x_{6}=\frac{\chi_{H} \chi_{I}}{\chi_{G}}
\end{array}\right.
$$

Then, the product-form condition required by $B B^{(2)}$, according to Theorem 1 , is (the product-form is unconditional for the other BBs): $\chi_{I}\left(\chi_{G}+\chi_{I}\right)=\chi_{A} \chi_{B}$. Under this assumption we have the following equilibrium probability distribution:

$$
\begin{aligned}
\pi(\mathbf{m}) \propto\left(\frac{\chi_{E} \chi_{H}^{2}\left(\chi_{G}+\chi_{I}\right)^{2}}{\chi_{D} \chi_{F} \chi_{G}^{2} \chi_{I}}\right)^{m_{1}} & \left(\frac{\chi_{F} \chi_{G} \chi_{I}}{\chi_{E} \chi_{H}\left(\chi_{G}+\chi_{I}\right)}\right)^{m_{2}}\left(\frac{\chi_{H}\left(\chi_{G}+\chi_{I}\right)}{\chi_{F} \chi_{G}}\right)^{m_{3}} \\
& \cdot\left(\frac{\chi_{I}}{\chi_{B}}\right)^{m_{4}}\left(\frac{\chi_{B} \chi_{H}}{\chi_{G} \chi_{I}}\right)^{m_{5}}\left(\frac{\chi_{H}\left(\chi_{G}+\chi_{I}\right)}{\chi_{G} \chi_{C}}\right)^{m_{6}}
\end{aligned}
$$

Example 3 (Open net unconditionally in product-form) In this example we analyse an open SPN whose product-form condition does not depend on the transition rates. The model is depicted by Figure 7-(A). Figure 7-(B) shows the (valid) net decomposition of the model, wherein the transitions are appropriately renamed and their rates shown in brackets; $\chi_{\alpha}$ is the rate of transition $T_{\alpha}$ of the original model $(\alpha=A, \ldots, I)$, and the rates denoted by $x_{*}$ are the unknown variables of the rate equations. We must solve the following non-linear 


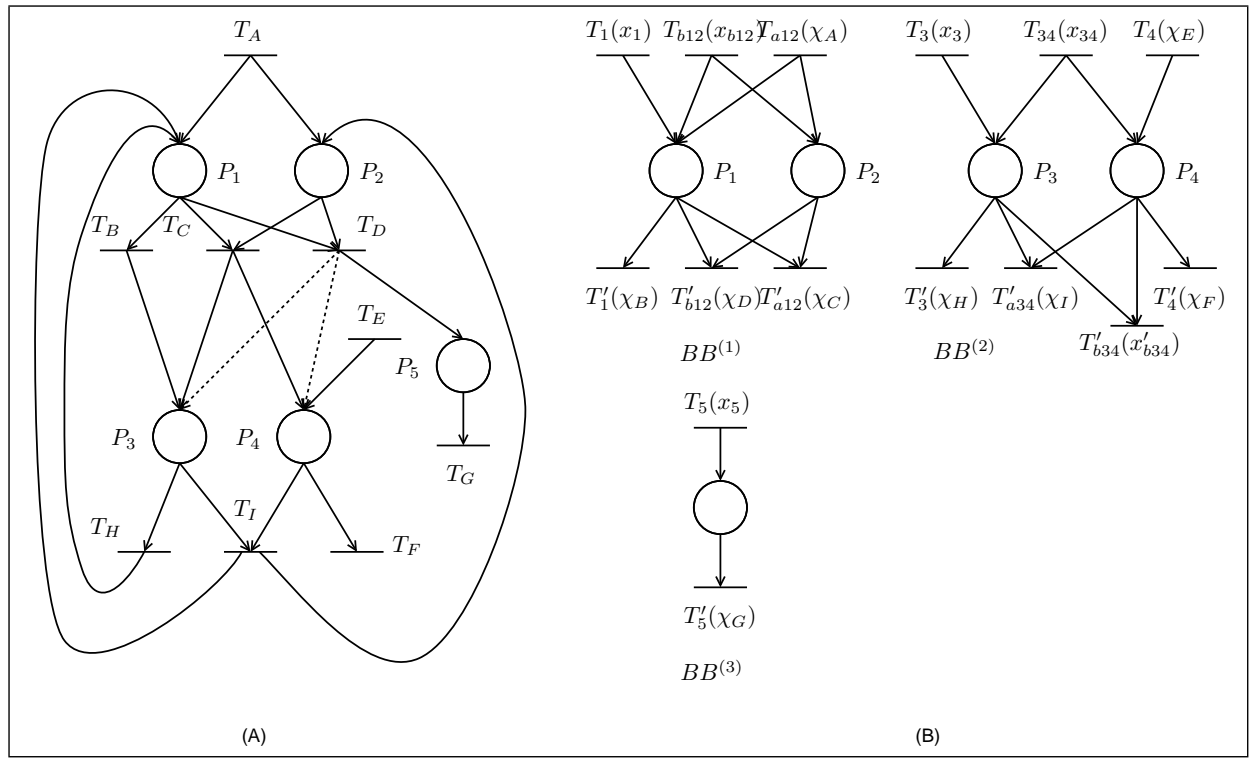

Figure 7: SPN of Example 3. (A) The original net. (B) The valid net decomposition.

system:

$$
\left\{\begin{array}{l}
x_{1}=x_{3} \\
x_{b 12}=x_{34} \frac{\chi_{I}}{\chi_{I}+x_{b 34}^{\prime}} \\
x_{3}=x_{1} \\
x_{34}=\left(\chi_{A}+x_{b 12}\right) \frac{\chi_{C}}{\chi_{C}+\chi_{D} \chi_{D}} \\
x_{b 34}^{\prime}=\left(\chi_{A}+x_{b 12}\right) \frac{\chi_{D}+\chi_{D}}{\chi_{C}} \\
x_{5}=x_{34} \frac{x_{b 34}^{\prime}}{\chi_{I}+x_{b 34}^{\prime}}
\end{array}\right.
$$

After routine algebra, let $\Delta=\chi_{A}^{2} \chi_{D}+2 \chi_{A} \chi_{I}\left(2 \chi_{C}+\chi_{D}\right)+\chi_{D} \chi_{I}^{2}$ and we have:

$$
\left\{\begin{array}{l}
x_{b 12}=\frac{1}{2}\left(-\chi_{A}-\chi_{I}+\sqrt{\frac{\Delta}{\chi_{D}}}\right) \\
x_{34}=\left(\chi_{A}-\chi_{I}+\sqrt{\frac{\Delta}{\chi_{D}}}\right) \frac{\chi_{C}}{2\left(\chi_{C}+\chi_{D}\right)} \\
x_{b 34}^{\prime}=\frac{\chi_{A} \chi_{D}-\chi_{D} \chi_{I}+\sqrt{\chi_{D} \Delta}}{2\left(\chi_{C}+\chi_{D}\right)} \\
x_{5}=\frac{\chi_{A}\left(2 \chi_{C}+\chi_{D}\right)+\chi_{D} \chi_{I}-\sqrt{\chi_{D} \Delta}}{2\left(\chi_{C}+\chi_{D}\right)} \\
x_{1}=x_{3}
\end{array}\right.
$$

As it stands, the rate equations do not have a unique solution for the unknowns $x_{1}$ and $x_{3}$. However, the product-form constraint of $B B^{(2)}$ gives, from Theorem 1:

$$
x_{34} \chi_{H} \chi_{F}=x_{3} \chi_{E}\left(\chi_{I}+\chi_{b 34}^{\prime}\right)
$$




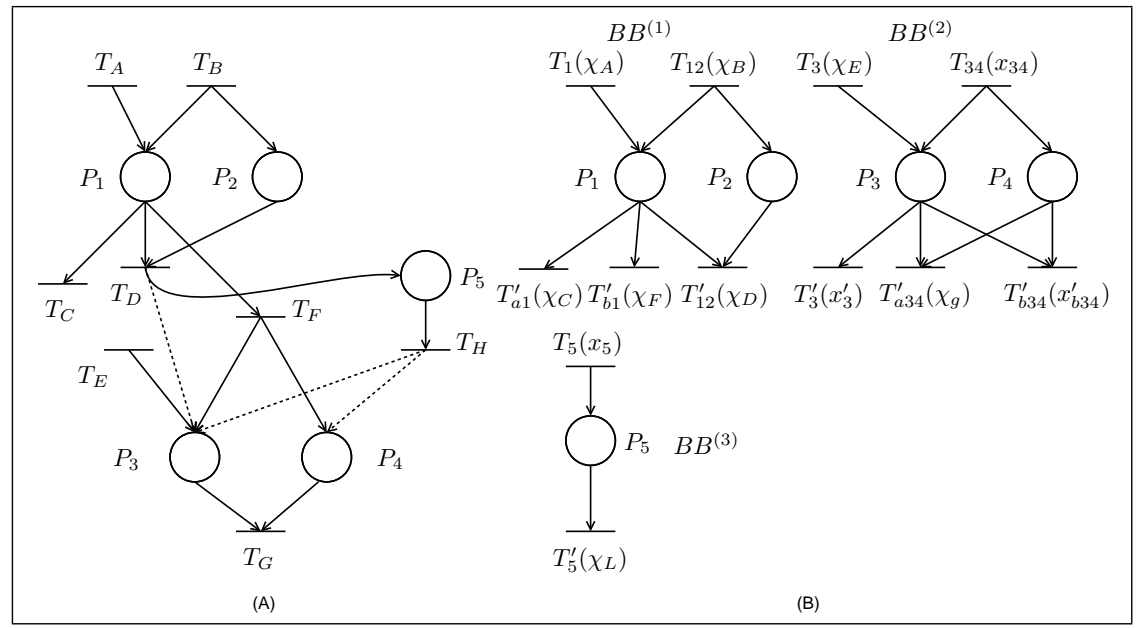

Figure 8: Open net with two trigger-transitions. Transition $T_{H}$ removes a token from $P_{3}$ and $P_{4}$ (if both are non-empty) without generating any other token, while $T_{D}$ transfers a token (if any is present) from $P_{3}$ to $P_{5}$.

Solving for $x_{3}$, gives:

$$
x_{3}=\left(-\chi_{A}-\chi_{I}+\sqrt{\frac{\Delta}{\chi_{D}}}\right) \frac{\chi_{F} \chi_{H}}{2 \chi_{E} \chi_{I}}
$$

$B B^{(1)}$ and $B B^{(3)}$ are unconditionally in product-form. Hence, applying Theorem 1 , we have the following $\rho$-values:

$$
\rho_{1}=\frac{x_{1}}{\chi_{B}}, \quad \rho_{2}=\frac{\chi_{A}+x_{b 12}}{\chi_{C}+\chi_{D}} \frac{\chi_{B}}{x_{1}}, \quad \rho_{3}=\frac{x_{3}}{\chi_{H}}, \quad \rho_{4}=\frac{\chi_{E}}{\chi_{F}}, \quad \rho_{5}=\frac{x_{5}}{\chi_{G}}
$$

and the unconditional steady-state distribution:

$$
\pi(\mathbf{m}) \propto \prod_{i=1}^{5} \rho_{i}^{m_{i}}
$$

Example 4 (Open net with negative tokens) In this example we show that it is possible to represent negative tokens by means of trigger-transitions in the same way that negative customers can be represented by means of triggers in G-networks. We consider the model of Figure 8-(A) in which the firing of transition $T_{H}$ removes two customers simultaneously from places $P_{4}$ and $P_{5}$, without generating any token. Figure 8-(B) shows the well-formed net decomposition. The system of rate equations is:

$$
\left\{\begin{array}{l}
x_{34}=\frac{\chi_{A} \chi_{F}}{\chi_{C}+\chi_{F}} \\
x_{3}^{\prime}=\chi_{B} \\
x_{b 34}^{\prime}=x_{5} \\
x_{5}=\chi_{E}
\end{array}\right.
$$


the solution of which is obvious. By Theorem $1, B B^{(1)}, B B^{(2)}, B B^{(3)}$ are unconditionally in product-form and by Theorem 2 we have:

$$
\begin{aligned}
& \pi(\mathbf{m}) \propto\left(\frac{\chi_{A}}{\chi_{C}+\chi_{F}}\right)^{m_{1}}\left(\frac{\chi_{B}\left(\chi_{C}+\chi_{F}\right)}{\chi_{A} \chi_{D}}\right)^{m_{2}}\left(\frac{\chi_{E}}{\chi_{B}}\right)^{m_{3}} \\
& \cdot\left(\frac{\chi_{A} \chi_{B} \chi_{F}}{\chi_{E}\left(\chi_{C}+\chi_{F}\right)\left(\chi_{E}+\chi_{G}\right)}\right)^{m_{4}}\left(\frac{\chi_{E}}{\chi_{H}}\right)^{m_{5}}
\end{aligned}
$$

\subsection{SPNs with catastrophes}

Queueing networks with catastrophes are a special type of G-networks, in which signals cause the destination queue to empty immediately; a product-form solution with non-linear traffic equations was obtained in $[6,10]$. For SPNs, we consider special transitions $T^{\bullet}$, the firings of which cause their output places (with non-zero components in the output vector $\mathbf{O}\left(T^{\bullet}\right)$ ) to instantaneously reduce their numbers of tokens by an amount $K \geq 0$, the minimum number of tokens that are present in the places of $\sigma\left(\mathbf{O}\left(T^{\bullet}\right)\right)$, i.e. $K=\min _{\mathbf{O}\left(T^{\bullet}\right)_{i}>0} m_{i}$.

Informally, we can view the effect of the firings of a catastrophe-transition as the maximum reduction of the numbers of tokens in the output places under the constraint that each place looses the same number of tokens. Clearly, at least one output place is left empty - those with the lowest token-count in the marking.

\subsubsection{Semantics}

Like ordinary transitions, a catastrophe-transition $T^{\bullet}$ is characterised by an input vector $\mathbf{I}\left(T^{\bullet}\right)$ and an output vector $\mathbf{O}\left(T^{\bullet}\right)$. $T^{\bullet}$ is enabled if $\mathbf{m}-\mathbf{I}\left(T^{\bullet}\right) \geq \mathbf{0}$ and the state reached after its firing is $\mathbf{m}-\mathbf{I}\left(T^{\bullet}\right)-\delta\left(\mathbf{m}, T^{\bullet}\right)$, where the function $\delta: \mathbb{N}^{N} \times \mathcal{T} \rightarrow \mathbb{N}^{N}$ is defined by:

$$
\delta\left(\mathbf{m}, T^{\bullet}\right)_{k}= \begin{cases}0 & \text { if } \mathbf{O}\left(T^{\bullet}\right)_{k}=0 \\ \min _{\mathbf{O}\left(T^{\bullet}\right)_{i}>0} m_{i} & \text { otherwise }\end{cases}
$$

for $1 \leq k \leq N$, where $N$ is the number of places in the net.

Figure 9-(A) shows the graphical representation of a catastrophe-transition (a solid bar with a filled circle). To the authors' best knowledge, synchronised catastrophes is a new topic for G-networks and, as we will prove in the analysis that follows, rate-dependent product-form conditions may arise.

\subsubsection{Analysis}

The derivation of product-forms for SPNs with catastrophe-transitions is again based on net decomposition into BBs. Consider a set of places $\{1, \ldots, N\}$ which structurally forms a BB and suppose that we add an input catastrophetransition $T_{y}^{\bullet}$, with $y \in 2^{\{1, \ldots, N\}} \backslash \emptyset$ representing the set of the indices of the places $P_{i}$ such that $\mathbf{O}\left(T^{\bullet}\right)_{i}>0$. Figure 9-(A) shows the catastrophe-transition of the BB only and Figure 9-(B) shows the unfolding of transition $T^{\bullet}$ in terms of trigger-transitions. Informally, we can see the grey part of the picture as a cycle which deletes iteratively tokens from the set of places $y$-involving correspondingly multiple applications of RCAT in deriving the product-form. Note that 


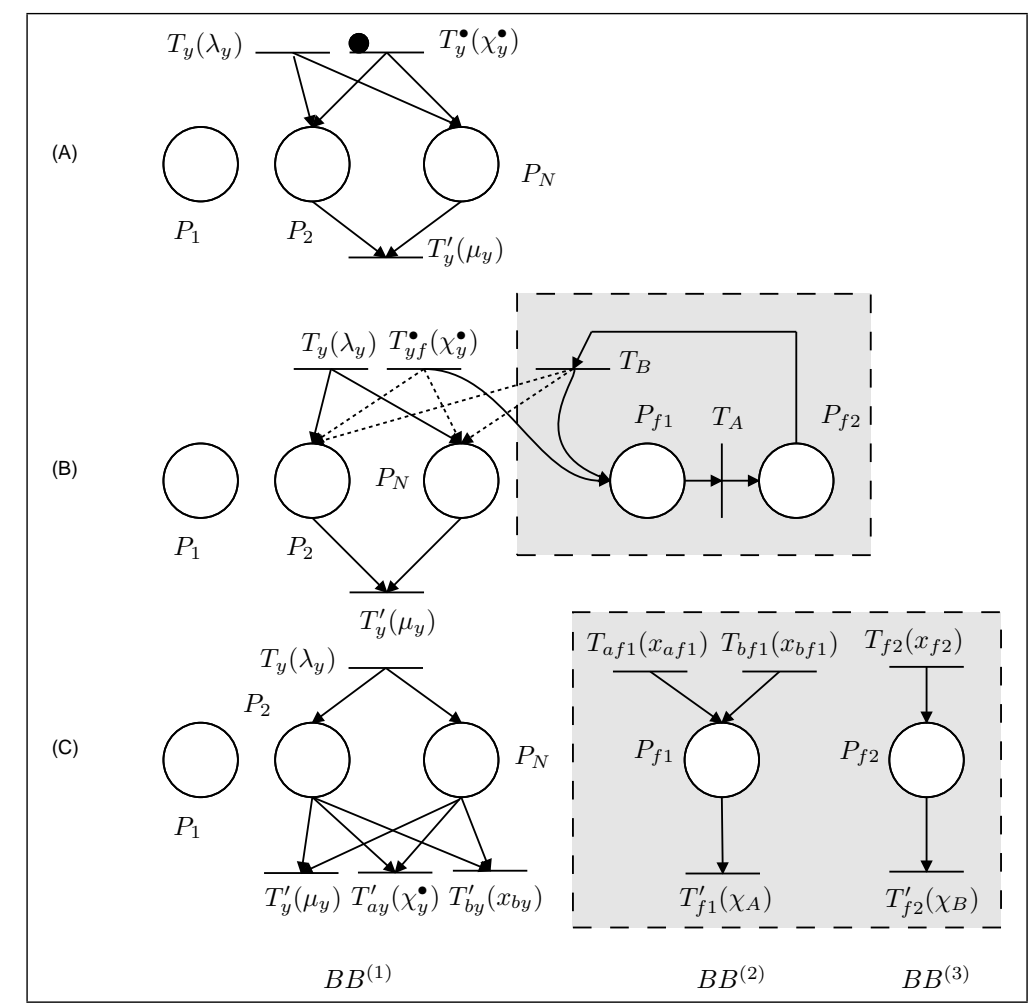

Figure 9: (A) Graphical representation of a catastrophe-transition. (B) Unfolding of a catastrophe-transition. (C) Decomposition into BBs.

the net of Figure 9-(B) is equivalent to that of Figure 9-(A) if transitions $T_{f 1}$ and $T_{f 2}$ are immediate (i.e., they fire in deterministically zero time). In the unfolded net $T_{f 1}^{\prime}$ and $T_{f 2}$ are associated with $T_{A} ; T_{b f 1}, T_{f 2}^{\prime}$ and $T_{b y}^{\prime}$ are associated with $T_{B}$; and, finally, $T_{a f 1}$ and $T_{a y}^{\prime}$ are associated with $T_{y f}^{\bullet}$. This net may now be studied with the technique introduced in Section 4. Figure 9-(C) shows the decomposition in BBs. Clearly $B B^{(2)}$ and $B B^{(3)}$ satisfy the conditions of Theorem 1, while $B B^{(1)}$ may present some rate-dependent product-form conditions. The rate equations are:

$$
\left\{\begin{array}{l}
x_{b y}=x_{f 2} \\
x_{a f 1}=\lambda_{y} \frac{\chi_{y}^{\bullet}}{\chi_{y}^{\bullet}+\mu_{y}+x_{b y}} \\
x_{b f 1}=\lambda_{y} \frac{x_{b y}}{\chi_{y}^{*}+\mu_{y}+x_{b y}} \\
x_{f 2}=x_{a f 1}+x_{b f 1}
\end{array}\right.
$$

We are especially interested in the solution for $x_{b y}$, which is the only unknown in $B B^{(1)}$. Its unique positive solution in the non-linear System (4) turns out to be:

$$
x_{b y}=\frac{1}{2}\left(\lambda_{y}-\chi_{y}^{\bullet}-\mu_{y}+\sqrt{\left(-\lambda_{y}+\chi_{y}^{\bullet}+\mu_{y}\right)^{2}+4 \lambda_{y} \chi_{y}^{\bullet}}\right) .
$$

It is worth noting that $x_{b y}$, and hence the steady-state distribution of $B B^{(1)}$, is independent of the rates of the auxiliary transitions $T_{A}$ and $T_{B}, \chi_{A}$ and $\chi_{B}$, 
respectively. Following the $\mathrm{BB}$ analysis and assuming $\lambda_{y}>0$ (while we may have $\mu_{y}=0$, i.e. $T_{y}$ may be missing) we have:

$$
\rho_{y}=\frac{\lambda_{y}}{\chi_{\dot{y}}^{\bullet}+\mu_{y}+x_{b y}}=\frac{\chi_{y}^{\bullet}+\mu_{y}+\lambda_{y}-\sqrt{\left(-\lambda_{y}+\chi_{\dot{y}}^{\bullet}+\mu_{y}\right)^{2}+4 \lambda_{y} \chi_{\dot{y}}^{\bullet}}}{2 \mu_{y}}
$$

and the product-form follows.

More generally, however, we can use this example to revise Definition 5 to incorporate catastrophe-transitions, as follows.

Definition 7 In a SPN with trigger-transitions and catastrophe-transitions, we say that two places $P_{i}$ and $P_{j} \in \mathcal{P}$ are directly connected with or without triggers and catastrophes, written $P_{i} \sim_{t c} P_{j}$, if and only if $P_{i} \sim_{t} P_{j}$, where a catastrophetransition is considered the same as an ordinary transition in the definition of $\sim_{t}$, i.e. the syntactic class of ordinary transitions is extended to include catastrophe-transitions.

We define the equivalence relation $\sim_{t c}^{*}$ to be the transitive closure of $\sim_{t c}$, and say that $P_{i}$ and $P_{j}$ are connected (with or without triggers and catastrophes) if $P_{i} \sim_{t c}^{*} P_{j}$.

The equivalence classes of $\sim_{t c}^{*}$ are labelled $\mathcal{C}^{(b)}, 1 \leq b \leq B$ where $B>0$ is the number of equivalence classes, superseding the previous definition which applied to ordinary and trigger-transitions.

Again proceeding as we did for trigger-transitions, we update Definition 6 to include catastrophe-transitions.

Definition 8 Given a SPN $S$ with trigger-transitions, catastrophe-transitions and $\sim_{t c}^{*}$-equivalence classes $\mathcal{C}^{(b)}, 1 \leq b \leq B$, the net decomposition of $S$ is the set of $S P N s S^{\text {dec }}=\left\{B B^{(b)}, 1 \leq b \leq B\right\}$, in which the set of places of $B B^{(b)}$ is $\mathcal{C}^{(b)}$ and the transitions of each $B B^{(b)}$ in $S^{\text {dec }}$ are given by the following, where the generic transition $T \in \mathcal{T}_{S}$ with rate $\chi$ is in $S$ :

- If $T$ is an ordinary or trigger-transition, the corresponding transitions given by Definition 6 all exist in the $B B^{(b)}$ s, with the same rate definitions.

- If $T$ is a catastrophe-transition, let $s=\sigma(\mathbf{I}(T)) \subseteq \mathcal{C}^{(f)}, s \neq \emptyset$ for some $f, 1 \leq f \leq B$. Then $B B^{(f)}$ has an output transition $T_{s}^{\prime}$ with $\mathbf{I}\left(T_{s}^{\prime}\right)=\mathbf{I}(T)$ and rate $\chi$.

- If $T$ is a catastrophe-transition, let $v=\sigma(\mathbf{O}(T)) \subseteq \mathcal{C}^{(g)}, v \neq \emptyset$ for some $g, 1 \leq g \leq B$. Then $B B^{(g)}$ has two output transitions, $T_{a v}^{\prime}$ and $T_{b v}^{\prime}$, with $\mathbf{I}\left(T_{a v}^{\prime}\right)=\mathbf{I}\left(T_{b v}^{\prime}\right)=\mathbf{O}(T)$ and respective rates $x_{a v}^{\bullet}$, which is equal to $\chi$ if $\mathbf{I}(T)=\mathbf{0}$ or to the reversed rate of $T_{u}^{\prime}$ in $B B^{(f)}$ (for some $f, 1 \leq f \leq B$ ), containing the places $u=\sigma(\mathbf{I}(T))$, otherwise and

$$
x_{b v}=\frac{1}{2}\left(\lambda_{v}-x_{a v}^{\bullet}-\mu_{v}+\sqrt{\left(-\lambda_{v}+x_{a v}^{\bullet}+\mu_{v}\right)^{2}+4 \lambda_{v} x_{a v}^{\bullet}}\right)
$$

where:

$-\lambda_{v}$ is sum of the rates of the input transitions in $B B^{(g)}$ for which the non-zero components of their output vectors are indexed by $v$, 


\section{- $\mu_{v}$ is the sum of the rates of the output transitions in $B B^{(g)}$ for which the non-zero components of their input vectors are indexed by $v$.}

$A$ net decomposition is said to be well-formed if $B B^{(i)}$ is a $B B$ for every $i=$ $1, \ldots, B$.

The following Theorem applies this definition to give conditions for productform in a SPN with trigger- and catastrophe-transitions and, if these are satisfied, its steady-state probability distribution.

Theorem 3 A SPN $S$ with well-formed decomposition $S^{d e c}=\left\{B B^{(1)}, \ldots, B B^{(B)}\right.$ has product-form solution:

$$
\pi(\mathbf{m}) \propto \prod_{b=1}^{N} \pi_{b}(\mathbf{m})
$$

where $\pi_{b}$ is an un-normalised product-form solution of $B B^{(b)}$, with rates set according to Definition 8, if each $B B^{(b)}$ satisfies the rate conditions of Theorem 1.

Proof. Given a SPN with signals, we can define a new SPN in which each catastrophe-transition is unfolded according to the schema depicted by Figure 9. We previously observed that the steady-state probability distribution of the numbers of tokens in the places at which the catastrophe-transitions operate is independent of the rates assigned to the fictitious transitions. The result follows by taking the limit to infinity of the fictitious transition rates, added in the unfolding of the catastrophe-transitions.

The following corollary is useful for simplifying the system of rate equations in the case of multiple catastrophe-transitions with the same output vector.

Corollary 1 Given a SPN with signal transitions, suppose that there exists a set $\mathcal{T}^{\bullet}=\left\{T_{i}^{\bullet}\right\}$ such that all $T_{i}^{\bullet}$ are catastrophe-transitions that share the same output vector $\mathbf{O}\left(\mathcal{T}^{\bullet}\right) \neq \mathbf{0}$. Let $B B^{(c)}$, with $v=\sigma\left(\mathbf{O}\left(\mathcal{T}^{\bullet}\right)\right) \subseteq \mathcal{C}^{(c)}$, be the $B B$ obtained by applying the decomposition of Definition 7 , where for each transition $T_{i}^{\bullet} \in \mathcal{T}^{\bullet}$ there is a pair of transitions $T_{a_{i} v}$ and $T_{b_{i} v}$ with rates $x_{a_{i} v}^{\bullet}$ and $x_{b_{i} v}$.

Then, the steady-state distribution of $B B^{(c)}$ does not change if all the transitions $T_{a_{i} v}$ and $T_{b_{i} v}$ are replaced by a single pair of transitions $T_{\alpha v}^{\prime}$, and $T_{\beta v}^{\prime}$ with $\mathbf{I}\left(T_{\alpha v}^{\prime}\right)=\mathbf{I}\left(T_{\beta v}^{\prime}\right)=\mathbf{O}\left(\mathcal{T}^{\bullet}\right)$ and with rates $x_{\alpha v}^{\bullet}=\sum_{T_{i}^{\bullet} \in \mathcal{T}} x_{a_{i} v}$ and

$$
x_{\beta v}=\frac{1}{2}\left(\lambda_{v}-x_{\alpha v}^{\bullet}-\mu_{v}+\sqrt{\left(-\lambda_{v}+x_{\alpha v}^{\bullet}+\mu_{v}\right)^{2}+4 \lambda_{v} x_{\alpha v}^{\bullet}}\right)
$$

Proof. The proof is straightforward by the observation that Equation (6) is obtained from equations (4). 


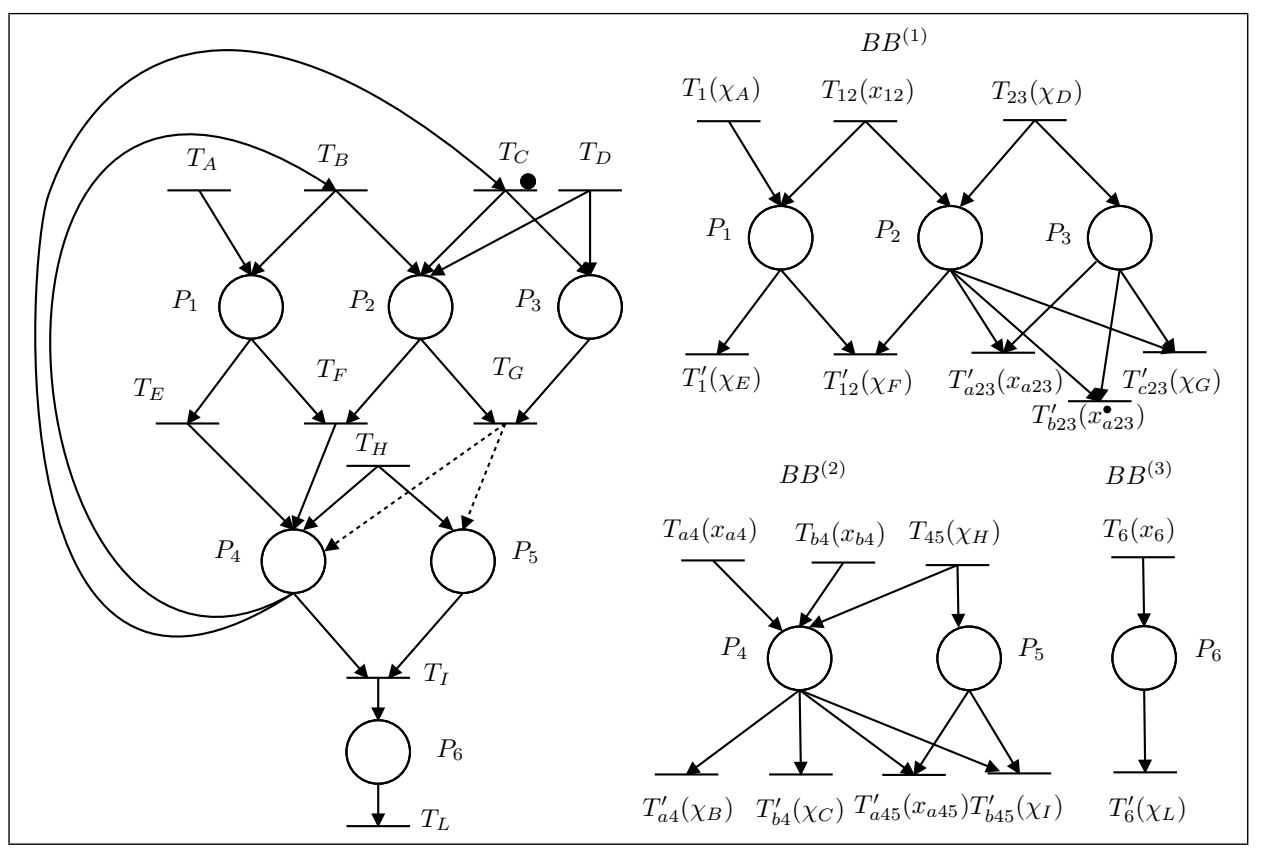

Figure 10: SPN of Example 5 with signals and its decomposition into BBs.

\subsubsection{Example}

We now show some applications of Theorem 3 that derive both rate-independent and rate-dependent product-form conditions for SPNs with trigger and catastrophetransitions.

Example 5 (SPN with unconditioned Product-form) In the SPN shown in Figure 10, with both trigger- and catastrophe-transitions, $T_{C}$ causes a synchronised catastrophe at places $P_{2}$ and $P_{3}$ and is modelled in the corresponding BB decomposition by transitions $T_{a 23}^{\prime}$ and $T_{b 4}^{\prime}$. Transition $T_{G}$ is a triggertransition, the firing of which causes the deletion of a token in $P_{4}$ and $P_{5}$. It is clear that the CTMC underlying this net is irreducible. Figure 10 illustrates the decomposition into BBs of the SPN. Note that the net decomposition itself ensures that the product-form will be unconditional because the rates of the input/output transitions of the BBs satisfy the conditions of Theorem 1.

According to Theorem 3, we can write down the following non-linear system of rate equations:

$$
\left\{\begin{array}{l}
x_{12}=\frac{x_{a 4}+x_{b 4} \chi_{B}}{\chi_{B}+\chi_{C}} \\
x_{a 23}=\frac{1}{2}\left(\chi_{D}-x_{b 23}^{\bullet}-\chi_{G}+\sqrt{\left(-\chi_{D}+x_{b 23}^{\bullet}+\chi_{G}\right)^{2}+4 \chi_{D} x_{b 23}^{\bullet}}\right) \\
x_{b 23}^{\bullet}=\frac{\left(x_{a 4}+x_{b 4}\right) \chi_{C}}{\chi_{B}+\chi_{C}} \\
x_{a 4}=\chi_{A} \\
x_{b 4}=x_{12} \\
x_{a 45}=\chi_{D} \frac{\chi_{G}}{x_{a 23}+\chi_{G}+x_{b 23}^{\bullet}} \\
x_{6}=\chi_{H} \frac{\chi_{I}}{\chi_{I}+x_{a 45}}
\end{array}\right.
$$

Let $\Delta=\left(\chi_{A}+\chi_{D}\right)^{2}+2\left(\chi_{A}-\chi_{D}\right) \chi_{G}+\chi_{G}^{2}$. Then the unique positive solution 
of the system is:

$$
\begin{aligned}
& x_{12}=\frac{\chi_{A} \chi_{B}}{\chi_{C}} \quad x_{a 23}=\frac{1}{2}\left(-\chi_{A}+\chi_{D}-\chi_{G}+\sqrt{\Delta}\right) \quad x_{a 4}=\lambda_{A} \\
& x_{b 4}=\frac{\chi_{A} \chi_{B}}{\chi_{C}} \quad x_{a 45}=\frac{1}{2}\left(\chi_{A}+\chi_{D}+\chi_{G}-\sqrt{\Delta}\right) \\
& x_{6}=\frac{2 \chi_{H} \chi_{I}}{\chi_{A}+\chi_{D}+\chi_{G}+2 \chi_{I}-\sqrt{\Delta}}
\end{aligned}
$$

Since $B B^{(1)}, B B^{(2)}$ and $B B^{(3)}$ are in product-form, we may compute the $\rho_{i}$ associated with each place in the net as:

$$
\begin{aligned}
& \rho_{1}=\frac{\chi_{A}}{\chi_{E}}, \quad \rho_{2}=\frac{\rho_{12}}{\rho_{1}}=\frac{\chi_{B} \chi_{E}}{\chi_{C} \chi_{F}} \\
& \rho_{3}=\frac{\rho_{23}}{\rho_{2}}=\frac{\chi_{D}}{x_{a 23}+\chi_{b 23}^{\bullet} \chi_{G}} \frac{1}{\rho_{2}}=\frac{2 \chi_{C} \chi_{D} \chi_{F}}{\chi_{B} \chi_{E}\left(\chi_{A}+\chi_{D}+\chi_{G}+\sqrt{\Delta}\right)} \\
& \rho_{4}=\frac{x_{a 4}+x_{b 4}}{\chi_{B}+\chi_{C}}=\frac{\chi_{A}}{\chi_{C}} \\
& \rho_{5}=\frac{\rho_{45}}{\rho_{4}}=\frac{\chi_{H}}{x_{a 45}+\chi_{I}} \frac{1}{\rho_{4}}=\frac{2 \chi_{C} \chi_{H}}{\chi_{A}\left(\chi_{A}+\chi_{D}+\chi_{G}+2 \chi_{I}-\sqrt{\Delta}\right)} \\
& \rho_{6}=\frac{x_{6}}{\chi_{L}}=\frac{2 \chi_{I}}{\chi_{L}\left(\chi_{A}+\chi_{D}+\chi_{G}+2 \chi_{I}-\sqrt{\Delta}\right)}
\end{aligned}
$$

This gives the product-form solution for the net:

$$
\pi(\mathbf{m}) \propto \prod_{i=1}^{6} \rho_{i}^{m_{i}} .
$$

Example 6 (Application of Corollary 1) In this example, we consider the net shown in Figure 11-(A). Transition $T_{\alpha}$ has rate $\chi_{\alpha}$ for $\alpha=A, \ldots, P$. The net has two catastrophe-transitions, $T_{C}^{\bullet}$ and $T_{D}^{\bullet}$ that share the same output vector. In this case, the application of Corollary 1 simplifies the solution of RCAT's rate equations. Indeed, by Corollary 1 , transitions $T_{C}^{\bullet}$ and $T_{D}^{\bullet}$ are unfolded in $B B^{(1)}$ into a pair of transitions $\left(T_{a 23}^{\prime}\right.$ and $\left.T_{b 23}^{\prime}\right)$ instead of two pairs as required by a straightforward application of Theorem 3. Given the decomposition into BBs shown in Figure 11-(B), the system of rate equations is:

$$
\left\{\begin{array}{l}
x_{123}=x_{4} \\
x_{a 23}^{\bullet}=\chi_{D}+\chi_{I} \\
x_{b 23}=\frac{1}{2}\left(\chi_{E}-x_{a 23}^{\bullet}-\chi_{G}+\sqrt{\left(\chi_{E}-x_{a 23}^{\bullet}-\chi_{G}\right)^{2}+4 \chi_{E} x_{a 23}^{\bullet}}\right) \\
x_{4}=x_{123} \quad \chi_{G} \\
x_{5}=\chi_{E} \frac{x_{a 23}^{\bullet}+x_{b 23}+\chi_{G}}{x_{56}=x_{7}} \\
x_{7}=x_{56} \quad\left(\text { Product-form condition of } B B^{(3)}\right) \\
\left.x_{56} \chi_{L} \chi_{C}=\chi_{M} x_{5} \chi_{I} \quad \text { (Product-form condition of } B B^{(1)}\right) \\
x_{123} \chi_{N}\left(x_{a 23}^{\bullet}+x_{b 23}+\chi_{G}\right)=\chi_{F} \chi_{A} \chi_{E} \quad \text { r }
\end{array}\right.
$$




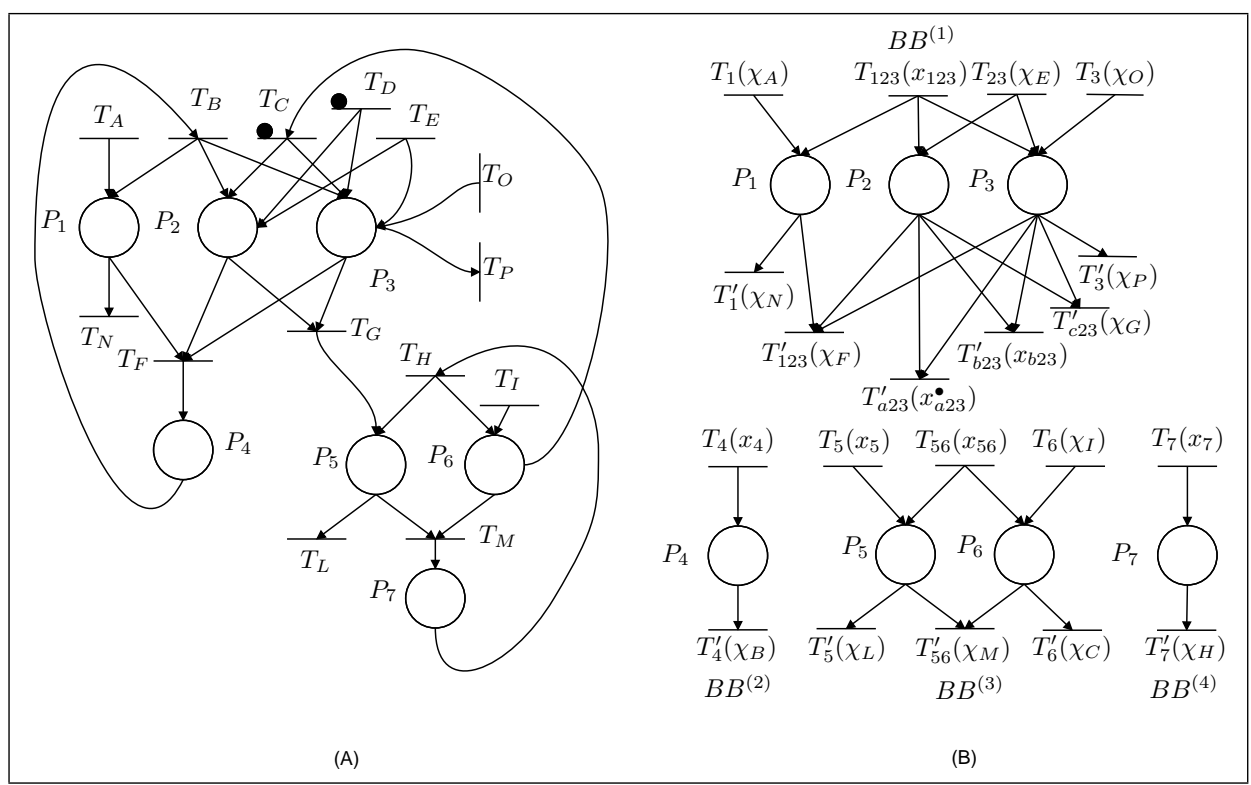

Figure 11: (A) SPN with catastrophes studied in Example 6. (B) Decomposition into BB.

The unique solution follows after some algebra: let

$$
\Delta=\left(\chi_{E}-\chi_{D}-\chi_{I}-\chi_{G}\right)^{2}+4 \chi_{E}\left(\chi_{D}+\chi_{I}\right),
$$

then:

$$
\begin{aligned}
& x_{123}=x_{4}=\frac{\chi_{A} \chi_{F}}{2 \chi_{G} \chi_{N}}\left(\chi_{D}+\chi_{E}+\chi_{G}+\chi_{I}-\sqrt{\Delta}\right) \\
& x_{a 23}=\chi_{D}+\chi_{I} \\
& x_{b 23}=\frac{1}{2}\left(\chi_{E}-\chi_{D}-\chi_{G}-\chi_{I}+\sqrt{\Delta}\right) \\
& x_{5}=\frac{1}{2}\left(\chi_{D}+\chi_{E}+\chi_{G}+\chi_{I}-\sqrt{\Delta}\right) \\
& x_{56}=x_{7}=\frac{\chi_{I} \chi_{M}}{2 \chi_{C} \chi_{L}}\left(\chi_{D}+\chi_{E}+\chi_{G}+\chi_{I}-\sqrt{\Delta}\right) .
\end{aligned}
$$

This solution of the rate-equations now allows us to write down the product-form solution:

$$
\pi(\mathbf{m}) \propto \prod_{i=1}^{7} \rho_{i}^{m_{i}}
$$


where the $\rho_{i}$ are obtained, in the usual way, as:

$$
\begin{aligned}
& \rho_{1}=\frac{\chi_{A}}{\chi_{N}}, \quad \rho_{2}=\frac{2 \chi_{E} \chi_{P}}{\chi_{O}\left(\chi_{E}+\chi_{D}+\chi_{G}+\chi_{I}+\sqrt{\Delta}\right)}, \quad \rho_{3}=\frac{\chi_{O}}{\chi_{P}}, \\
& \rho_{4}=\frac{\chi_{A} \chi_{F}}{2 \chi_{B} \chi_{G} \chi_{N}}\left(\chi_{D}+\chi_{E}+\chi_{G}+\chi_{I}-\sqrt{\Delta}\right), \\
& \rho_{5}=\frac{1}{2 \chi_{L}}\left(\chi_{D}+\chi_{E}+\chi_{G}+\chi_{I}-\sqrt{\Delta}\right), \quad \rho_{6}=\frac{\chi_{I}}{\chi_{C}}, \\
& \rho_{7}=\frac{\chi_{I} \chi_{M}}{2 \chi_{C} \chi_{H} \chi_{L}}\left(\chi_{D}+\chi_{E}+\chi_{G}+\chi_{I}-\sqrt{\Delta}\right) .
\end{aligned}
$$

Note that, without applying Corollary 1 , the net decomposition would be that depicted by Figure 12, where $T_{a 23}, T_{b 23}$ are associated with $T_{C}$, and $T_{c 23}, T_{d 23}$ are associated with $T_{D}$. The corresponding system of rate equations is now:

$$
\left\{\begin{aligned}
& x_{123}= x_{4} \\
& x_{a 23}^{\bullet}= \chi_{I} \\
& x_{b 23}= \frac{1}{2}\left(\chi_{E}-x_{c 23}^{\bullet}-x_{d 23}-\chi_{G}-x_{a 23}^{\bullet}\right. \\
&\left.+\sqrt{\left(\chi_{E}-x_{c 23}^{\bullet}-x_{d 23}-\chi_{G}-x_{a 23}^{\bullet}\right)^{2}+4 \chi_{E} x_{a 23}^{\bullet}}\right) \\
& x_{c 23}^{\bullet}= \chi_{D} \\
& x_{d 23}= \frac{1}{2}\left(\chi_{E}-x_{a 23}^{\bullet}-x_{b 23}-\chi_{G}-x_{c 23}^{\bullet}\right. \\
&\left.+\sqrt{\left(\chi_{E}-x_{a 23}^{\bullet}-x_{b 23}-\chi_{G}-x_{c 23}^{\bullet}\right)^{2}+4 \chi_{E} x_{c 23}^{\bullet}}\right) \\
& x_{4}= x_{123} \quad \chi_{G} \\
& x_{5}= \chi_{E} \frac{x_{a 23}+x_{b 23}+x_{c 23}^{\bullet}+x_{d 23}+\chi_{G}}{x_{56}=} \\
& x_{7} x_{7}=x_{56} \\
& x_{56} \chi_{L} \chi_{C}=\chi_{M} x_{5} \chi_{I} \\
& x_{123} \chi_{N}\left(x_{a 23}^{\bullet}+x_{b 23}+x_{c 23}^{\bullet}+x_{d 23}+\chi_{G}\right)=\chi_{F} \chi_{A} \chi_{E} .
\end{aligned}\right.
$$

\section{Conclusion}

We have shown that a class of SPNs with special transitions, called signaltransitions, has product-form solution. Signal-transitions, like ordinary transitions, are enabled if none out of a specified set of input-places is empty, and their firing has one of the following effects on the net:

- Trigger-transition: one token is removed from each of a specified a set of output-places and, if this operation can be done, one token is added to each of another set of places;

- Catastrophe-transition: the number of tokens at each of a specified set of places is decreased by the same amount, such that the place with the lowest population becomes empty.

Although the transitions in our model do not have an intrinsic probabilistic behaviour analogous to those in $[31,8]$, in which the output vector is chosen probabilistically, the use of multiple transitions with the same input vector allows 


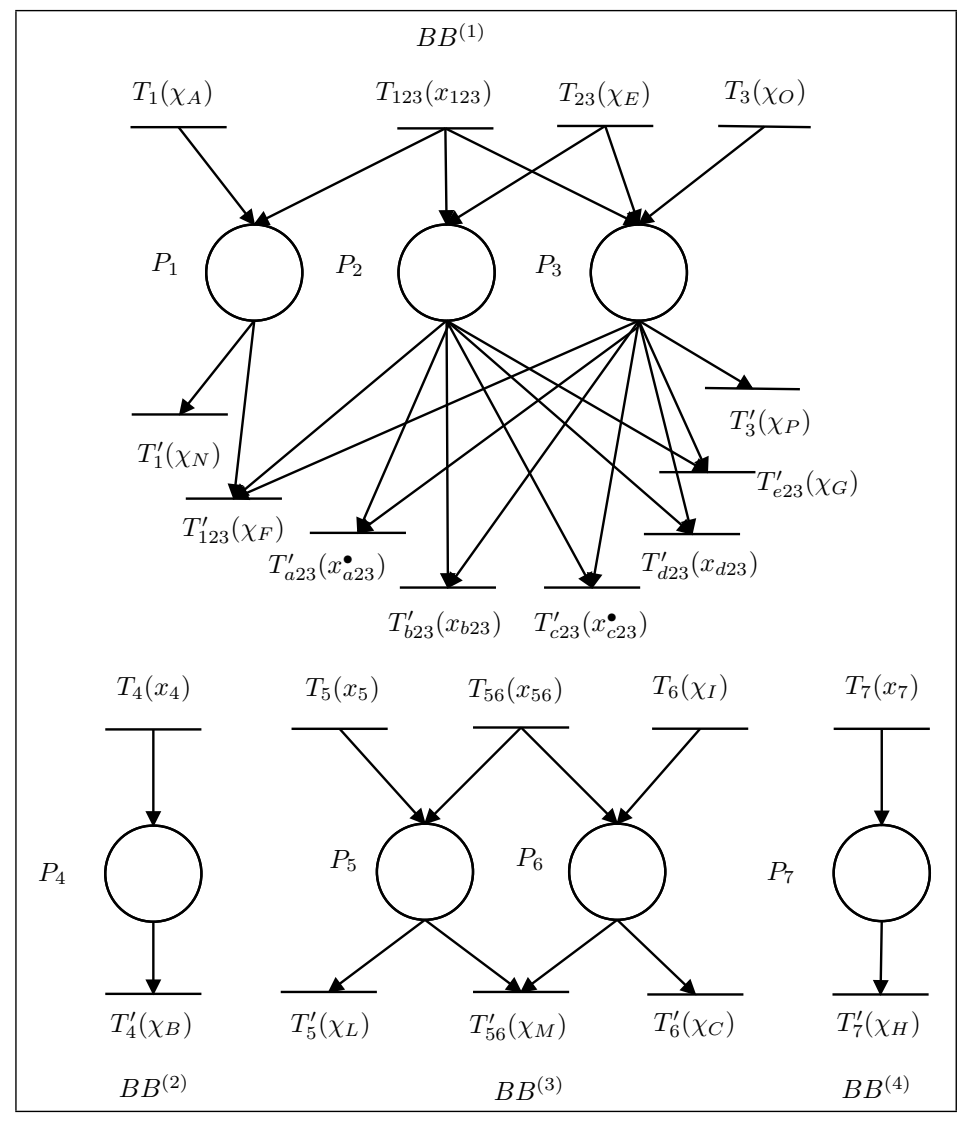

Figure 12: Decomposition of the net of Figure 11-(A) obtained without the application of Corollary 1.

one to obtain the same result, thanks to the standard properties of the minimum of independent, exponentially-distributed random variables. Our analysis method relies on a decomposition of a class of Petri nets into special structures, called building blocks (BBs). In general, the conditions for a SPN to belong to this class are not only structural (as with the product-form class studied in [26], for example) but also rate-dependent. In [31, 8], a class of SPN models with rate-dependent product-form conditions was studied, yielding a result based on a rank-theorem, in which the rank of a matrix whose entries depend on the whole net structure and the rates must be investigated. In our case, the rate-conditions are easier to test and have a clear interpretation because they are defined modularly for each BB - a net with a simple structure. Several examples of nets, with or without rate-dependent product-form conditions, have been presented to support these claims.

We derived the product-form solutions by means of RCAT [28] and the strength of this approach is twofold. First, we do not need to work with complicated systems of global balance equations, as most other approaches do. Secondly, we observe that this enhances the compositionality properties of the models that we analyse. For example, a transition whose reversed rate is con- 
stant (see Corollary 1) may be synchronised (as active) with any other model satisfying RCAT's conditions on passive transitions: the product-form of the whole model is then immediately guaranteed. If we interpret, as intuition suggests, the places of a SPN with signals as exponential queues, and the tokens as network customers, we can straightforwardly establish a product-form for a SPN with signals synchronised with any BCMP queueing nodes - even in the extended models proposed after the seminal BCMP paper, e.g. [34]. Future research directions involve the introduction of batch token movements and the development of efficient numerical techniques for the solution of the systems of non-linear rate equations that are required by this approach, and by all other G-network analyses.

\section{References}

[1] Ajmone Marsan, M., Balbo, G., and Conte, G. Performance Models of Microprocessor Systems. MIT Press, 1986.

[2] Artalejo, J. R. G-networks: A versatile approach for work removal in queueing networks. Eur. J. of Op. Res. 126, 2 (2000), 233-249.

[3] Balsamo, S., Harrison, P. G., And Marin, A. Methodological Construction of Product-form Stochastic Petri-Nets for Performance Evaluation. J. of System and Software (2012), To appear.

[4] Baskett, F., Chandy, K. M., Muntz, R. R., and Palacios, F. G. Open, closed, and mixed networks of queues with different classes of customers. J. ACM 22, 2 (1975), 248-260.

[5] Brouwer, L. E. J. Ueber eineindeutige, stetige transformationen von flächen in sich. Math. Ann. 69 (1910), 176-180.

[6] Chaо, X. A queueing network model with catastrophes and product form solution. Op. Res. Letters 18, 2 (1995), 75-79.

[7] Chao, X., Miyazawa, M., And Pinedo, M. Queueing networks: customers, signals and product form solutions. Wiley, 1999.

[8] Coleman, J. L., Henderson, W., and Taylor, P. G. Product form equilibrium distributions and a convolution algorithm for Stochastic Petri nets. Perf. Eval. 26, 3 (1996), 159-180.

[9] Dao-Thi, T., Fourneau, J. M., and Tran, M. G-networks with synchronised arrivals. Performance Evaluation 68, 4 (2011), 309-319.

[10] Fournau, J. M., Kloul, L., And Quessette, F. G-networks with jumps back to zero. In Proc. of ACM/IEEE MASCOTS (Duram, NC, USA, 1995), pp. 28-32.

[11] Fourneau, J. M., Gelenbe, E., And Suros, R. G-Networks with multiple classes of positive and negative customers. Theoret. Comput. Sci. 155 (1996), 141-156. 
[12] Fourneau, J. M., And Quessette, F. Computing the steady-state distribution of G-networks with synchronized partial flushing. In ISCIS, 21th International Symposium (Istanbul, Turkey, 2006), pp. 887-896.

[13] Gelenbe, E. Random neural networks with negative and positive signals and product form solution. Neural Computation 1, 4 (1989), 502-510.

[14] Gelenbe, E. Product form networks with negative and positive customers. J. of Appl. Prob. 28, 3 (1991), 656-663.

[15] Gelenbe, E. G-Networks with signals and batch removal. Prob. in the Eng. and Informational Sciences 7 (1993), 335-342.

[16] Gelenbe, E. G-networks with triggered customer movement. J. of Appl. Prob. 30 (1993), 742-748.

[17] Gelenbe, E. The first decade of G-Networks. E. J. of Op. Res. 126 (2000), 231-232.

[18] Gelenbe, E. G-networks: a unifying model for neural and queueing networks. Annals of Operations Research 48, 5 (October, 1994), 433-461.

[19] Gelenbe, E., and Fourneau, J. M. G-networks with resets. Perf. Eval. 49, 1-4 (2002), 179-191.

[20] Gelenbe, E., And Fourneau, J. M. Flow equivalence and stochastic equivalence in G-Networks. Comp. Management Sci. 1, 2 (2004), 179-192.

[21] Gelenbe, E., And Labed, A. G-Networks with multiple classes of signals and positive customers. E. J. of Op. Res. 48, 5 (1998), 293-305.

[22] Gelenbe, E., And Mitrani, I. Analysis and Synthesis of Computer Systems. Imperial College Press, 2010.

[23] Gelenbe, E., And Pujolle, G. Introduction to queueing networks. Wiley, 1998.

[24] Gelenbe, E., And Schassberger, M. Stability of product form GNetworks. Prob. in the Eng. and Informational Sciences 6 (1992), 271-276.

[25] Gelenbe, E., And Shachnai, H. On G-Networks and resource allocation in multimedia systems. E. J. of Op. Res. 126, 2 (2000), 308-318.

[26] Haddad, S., Moreaux, P., Sereno, M., and Silva, M. Product-form and stochastic Petri nets: a structural approach. Perf. Eval. 59, 4 (2005), $313-336$.

[27] Harrison, P., Patel, N., and Pitel, E. Reliability modelling using g-queues. E. J. of Op. Res. 126, 2 (2000), 273-287.

[28] Harrison, P. G. Turning back time in Markovian process algebra. Theoretical Computer Science 290, 3 (2003), 1947-1986.

[29] Harrison, P. G. Compositional reversed Markov processes, with applications to G-networks. Perf. Eval., Elsevier 57, 3 (2004), 379-408. 
[30] Harrison, P. G., And LeE, T. T. Separable equilibrium state probabilities via time reversal in Markovian process algebra. Theoretical Computer Science 346, 1 (2005), 161-182.

[31] Henderson, W., Lucic, D., and Taylor, P. G. A net level performance analysis of Stochastic Petri Nets. J. Austral. Math. Soc. Ser. B 31 (1989), 176-187.

[32] JACKsOn, J. R. Jobshop-like queueing systems. Management Science 10 (1963), 131-142.

[33] Kelly, F. Reversibility and stochastic networks. Wiley, New York, 1979.

[34] LE Boudec, J. Y. A BCMP extension to multiserver stations with concurrent classes of customers. In SIGMETRICS '86/PERFORMANCE '86: Proc. of the 1986 ACM SIGMETRICS Int. Conf. on Computer performance modelling, measurement and evaluation (New York, NY, 1986), ACM Press, pp. 78-91.

[35] Mairesse, J., And Nguyen, H. T. Deficiency Zero Petri Nets and Product Form. In Proc. of the 30th Int. Conf. on App. and Theory of Petri Nets (Paris, France, 2009), PETRI NETS '09, Springer-Verlag, pp. 103-122.

\section{A Salient features of ERCAT}

When two transitions in different Markov chains synchronise, as in the present description of $\mathrm{BBs}$, we consider one of them to be active, with a rate that becomes the rate of the joint transition (e.g., active action $t_{12}$ has rate $\lambda_{12}$ in $P^{1}$ ), and the other to be passive, with unspecified rate (usually denoted by a "top" symbol, e.g. $T_{12}$ for passive action $t_{12}$ in process $P^{2}$ ). The sets of outgoing, incoming, active and passive actions in each joint state are denoted according to the following notation (wherein $k=1,2$ ):

$\mathcal{P}_{k}$ : the set of passive action types in the process $P^{k}$ - each must be passive at all its instances in $P^{k}$;

$\mathcal{A}_{k}$ : the set of active action types in $P^{k}$ - each must be active at all its instances in $P^{k}$;

$\mathcal{P}^{(i, j) \rightarrow}$ : the set of action types that are passive and correspond to transitions out of joint state $(i, j)$ in the synchronisation;

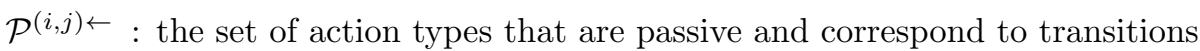
into joint state $(i, j)$ in the synchronisation;

$\mathcal{A}^{(i, j) \rightarrow}$ : the set of action types that are active and correspond to transitions out of joint state $(i, j)$ in the synchronisation;

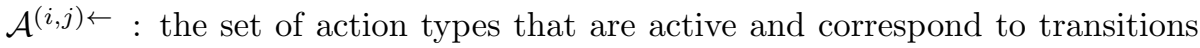
into joint state $(i, j)$ in the synchronisation;

$\alpha_{a}(i, j)$ : the instantaneous transition rate out of state $(i, j)$ corresponding to active action type $a$; 
$\beta_{a}(i, j)$ : the instantaneous transition rate out of state $(i, j)$ in the reversed joint Markov process, corresponding to passive action type $a$, with rate set to $x_{a}$ in one of the forwards component-processes (in which passive $a$ is therefore incoming to one of the local states $i$ or $j$.

Under appropriate conditions, a product-form is given by the following theorem, which is a simplified version of the more general result of [30] that derives the reversed process (and so product-form) of pairwise synchronisations amongst any finite number of processes. Prior to application of the theorem, in each component-process, every unspecified rate $T_{a}$ of a passive action with type $a$ is replaced by $x_{a}$-i.e. we replace $\left(a, \top_{a}\right)$ by $\left(a, x_{a}\right)$.

Theorem 4 (ERCAT) Suppose the following conditions hold in a synchronisation between two processes over the set of action types $L=\mathcal{P}_{1} \cup \mathcal{P}_{2}=\mathcal{A}_{1} \cup \mathcal{A}_{2}$ :

1. Every instance of a reversed action with type $\bar{a}$, of a forwards active action of type $a \in \mathcal{A}_{k}$, has the same rate $\overline{r_{a}}$ and $\left\{x_{a}\right\}$ satisfy the rate equations

$$
\left\{x_{a}=\overline{r_{a}} \mid a \in \mathcal{A}_{k}, 1 \leq k \leq n\right\} .
$$

2. The forward and reversed passive and active transition rates satisfy

$$
\begin{aligned}
& \sum_{a \in \mathcal{P}^{(i, j) \rightarrow}} x_{a}-\sum_{a \in \mathcal{A}^{(i, j) \leftarrow}} x_{a}=\sum_{a \in \mathcal{P}^{(i, j) \leftarrow} \backslash \mathcal{A}^{(i, j) \leftarrow}} \beta_{a}(i, j) \\
& -\sum_{a \in \mathcal{A}^{(i, j) \rightarrow \backslash \mathcal{P}^{(i, j) \rightarrow}}} \alpha_{a}(i, j) .
\end{aligned}
$$

Then there is a product-form solution for the equilibrium state probabilities (when these exist), which is $\pi(i, j) \propto \pi_{1}(i) \pi_{2}(j)$ where $\pi_{k}($.$) is the equilibrium$ probability function for the process $P_{k}, k=1,2$.

To apply the theorem it is important to be able to calculate the reversed rate of a transition. This is straightforward in any stationary Markov process in which the forward rate and the stationary state probabilities are known. Suppose a transition from state $i$ to state $j$ has rate $\lambda$. Then the reversed rate is (see [33], for example):

$$
\bar{\lambda}=\frac{\pi(i) \lambda}{\pi(j)}
$$

\section{A.1 Application of ERCAT to the basic BB}

We first consider the constraints on the transition rates imposed by the second condition of ERCAT. Note that the outgoing and incoming transitions and rates are the same for all states $P_{k}^{1}$ and $P_{k}^{2}$ with $k>0$. Therefore, it is only necessary to analyse the four joint states $(0,0),(0, k),(k, k),(k, 0)$ for some $k>0$ :

- $(0,0)$. For this state we have the following sets:

$$
\begin{aligned}
& \mathcal{P}^{(0,0) \rightarrow}=\left\{t_{12}\right\} \quad \mathcal{A}^{(0,0) \leftarrow}=\left\{t_{12}^{\prime}\right\} \\
& \mathcal{P}^{(0,0) \leftarrow} \backslash \mathcal{A}^{(0,0) \leftarrow}=\emptyset \quad \mathcal{A}^{(0,0) \rightarrow} \backslash \mathcal{P}^{(0,0) \rightarrow}=\emptyset .
\end{aligned}
$$

In order to apply ERCAT, Condition 2 must be satisfied and so we require $x_{12}=x_{12}^{\prime}$. 
- $(0, k)$. The corresponding sets for this state are:

$$
\begin{aligned}
& \mathcal{P}^{(0, k) \rightarrow}=\left\{t_{12}\right\} \quad \mathcal{A}^{(0, k) \leftarrow=\left\{t_{12}^{\prime}\right\}} \\
& \mathcal{P}^{(0, k) \leftarrow} \backslash \mathcal{A}^{(0, k) \leftarrow}=\left\{t_{12}, t_{12}^{\prime}\right\} \backslash\left\{t_{12}^{\prime}\right\}=\left\{t_{12}\right\} \\
& \mathcal{A}^{(0, k) \rightarrow} \backslash \mathcal{P}^{(0, k) \rightarrow}=\left\{t_{12}, t_{12}^{\prime}\right\} \backslash\left\{t_{12}\right\}=\left\{t_{12}^{\prime}\right\} \text {. }
\end{aligned}
$$

To satisfy Condition 2, we require

$$
x_{12}-x_{12}^{\prime}=\beta_{12}(0 k)-\alpha_{12}^{\prime}(0 k)
$$

where we use the abbreviations $\alpha_{12}(0 k) \equiv \alpha_{t_{12}}(0 k)$ and $\alpha_{12}^{\prime}(0 k) \equiv \alpha_{t_{12}^{\prime}}(0 k)$ and similarly for $\beta, \beta^{\prime}$. But since $x_{12}=x_{12}^{\prime}$, we conclude $\beta_{12}(0 k)=$ $\alpha_{12}^{\prime}(0 k)=\mu_{12}, t_{12}^{\prime}$ being active in process $P^{2}$.

- $(k, 0)$ and $(k, k)$. For these states the ERCAT Condition 2 is trivially satisfied since $\mathcal{P}^{(k, 0) \rightarrow}=\mathcal{A}^{(k, 0) \leftarrow}=\mathcal{P}^{(k, k) \rightarrow}=\mathcal{A}^{(k, k) \leftarrow}=L$. In fact, every passive action and every reversed action corresponding to an active action are always enabled, so that for these states the original RCAT structural conditions are satisfied [28].

The rate equations (Condition 1) for this model require, using Equation (7),

$$
\left\{\begin{array}{l}
x_{12}=\frac{\lambda_{12}\left(\mu_{1}+x_{12}^{\prime}\right)}{\lambda_{12}+\lambda_{1}} \\
x_{12}^{\prime}=\frac{\mu_{12}\left(\lambda_{2}+x_{12}\right)}{\mu_{12}+\mu_{2}}
\end{array} .\right.
$$

Since $x_{12}=x_{12}^{\prime}$, we obtain:

$$
\left\{\begin{array}{l}
x_{12}=\frac{\lambda_{12} \mu_{1}}{\lambda_{1}} \\
x_{12}^{\prime}=\frac{\mu_{12} \lambda_{2}}{\mu_{2}}
\end{array}\right.
$$

which gives the condition:

$$
\lambda_{1} \lambda_{2} \mu_{12}=\lambda_{12} \mu_{1} \mu_{2}
$$

Finally, we verify that

$$
\beta_{12}(0 k)=\frac{x_{12}\left(\mu_{2}+\mu_{12}\right)}{x_{12}+\lambda_{2}}=\mu_{12}
$$

as required.

To sum up, when Condition (8) is satisfied, the steady-state probability function $\pi\left(m_{1}, m_{2}\right)$ is in product-form. Moreover, the Markov processes associated with $P^{1}$ and $P^{2}$ are simple birth-death processes with equilibrium probabilities (when they exist) proportional to

$$
\left(\frac{\lambda_{1}+\lambda_{12}}{\mu_{1}+x_{12}^{\prime}}\right)^{m_{1}} \text { and }\left(\frac{\lambda_{2}+x_{12}}{\mu_{2}+\mu_{12}}\right)^{m_{2}} .
$$

Given the above conditions, the joint stationary probabilities simplify to

$$
\pi\left(m_{1}, m_{2}\right) \propto\left(\frac{\lambda_{1}}{\mu_{1}}\right)^{m_{1}}\left(\frac{\lambda_{2}}{\mu_{2}}\right)^{m_{2}}
$$




\section{B Technical contribution of this paper compared with previously published work}

We provide this comparison so as to give an interpretation in terms of GNetworks for readers that may be less comfortable with product-forms in stochastic Petri nets. We start by considering the work of [16], in which it is noted that the introduction of triggering signals in G-networks allows one to model the effect of token movements in Petri nets. Indeed, at a trigger arrival epoch, three nodes may change their states instantaneously: the one from which it departs as a signal, the one it arrives at, loosing a customer and, finally, the node that accepts the transferred customer. With respect to this behaviour our results allow one to model:

- synchronised customer arrivals at a set of queues;

- synchronised service completions at a set of queues;

- a signal that can remove one customer from a set of queues if they are all non-empty (instead of a single one);

- the effect of a signal adding a customer to a set of queues (instead of a single one).

As an example, let us consider a system consisting of four nodes $Q_{i}$ with $i=1, \ldots, 4$. Customers arrive from the outside at queue $Q_{i}$ with rate $\lambda_{i}$. At a service completion instant at $Q_{1}$, one customer is added to both $Q_{2}$ and $Q_{3}$ simultaneously with probability $p$. On a service completion at $Q_{2}$ or $Q_{3}$, a customer is sent to $Q_{4}$. When $Q_{4}$ completes a service, the customer leaves the system. With probability $1-p$ a customer leaving $Q_{1}$ arrives at $Q_{2}$ and $Q_{3}$ as a trigger, whereupon, if both $Q_{2}$ and $Q_{3}$ are non empty, a customer is removed from each of them and is added to $Q_{4}$. Figure 13 illustrates this system.

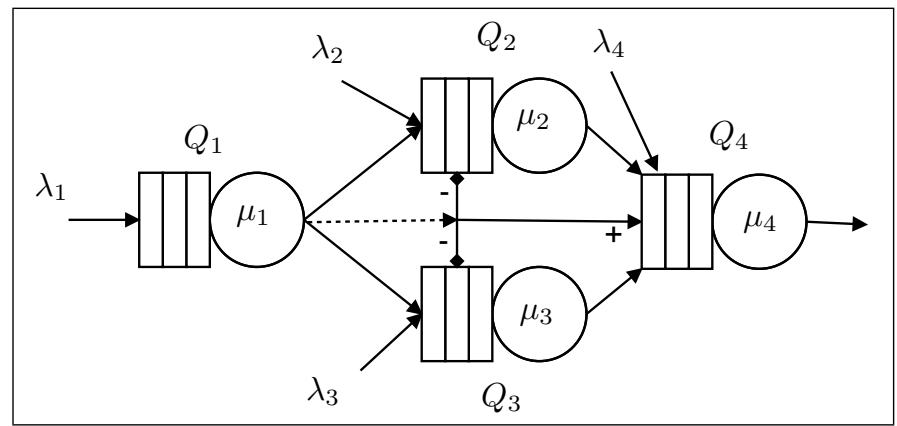

Figure 13: Model with instantaneous trigger propagation. The dashed line represents the customer leaving $Q_{1}$ as a signal and arriving at $Q_{2}$ and $Q_{3}$, simultaneously. If both the queues are non empty then a customer is immediately removed from each of them, and one is added to $Q_{4}$. Observe that the two solid lines from $Q_{1}$ to $Q_{2}$ and $Q_{3}$ denote a simultaneous customer arrival at the latter queues and not a probabilistic routing.

The model clearly differs from other G-networks because of the synchronised arrival at $Q_{2}$ and $Q_{3}$ on a service completion in $Q_{1}$. Moreover, observe that, in 
addition, the customer deletion policy at $Q_{2}$ and $Q_{3}$ is different from what is proposed in, for instance in [12, 29]. In fact, in these papers the signal would first arrive at $Q_{2}\left(Q_{3}\right)$ and, if this were not empty, would go to $Q_{3}\left(Q_{2}\right)$. If $Q_{3}$ were also not empty, then a customer would finally be added to $Q_{4}$. Now assume that $Q_{2}$ has $n_{2}>0$ customers and that $Q_{3}$ is empty. According to the deletion policy of the model of Figure 13, the signal arriving at $Q_{2}$ and $Q_{3}$ vanishes. Conversely, if we assume the simultaneous deletion policy described in $[12,29]$ with $Q_{2}$ reached before $Q_{3}$, then the signal deletes one customer from $Q_{2}$ before vanishing on arrival at $Q_{3}$. This different behaviour, combined with the synchronised positive customer arrivals at $Q_{2}$ and $Q_{3}$, lead to different productform conditions and expressions. In practice, we can show that a product-form solution holds if $p \lambda_{1} \mu_{2} \mu_{3}=(1-p) \lambda_{1} \lambda_{2} \lambda_{3}$. As shown in [3], if this condition is not satisfied, a product-form stationary distribution for $Q_{2}$ and $Q_{3}$ does not exist in general. Observe that, in general, product-forms derived for G-networks do not have rate-dependent conditions as do those derived in this simple case. 